\title{
First Total Synthesis of Mycothiol and Mycothiol-disulfide
}

\author{
Sungwon Lee and John P. N. Rosazza* \\ Division of Medicinal and Natural Products Chemistry, College of Pharmacy, and Center for \\ Biocatalysis and Bioprocessing, The University of Iowa, Iowa City, Iowa 52242
}

\section{Supporting Information}

\section{General Experimental}

All moisture-sensitive reactions were carried out under a positive pressure of nitrogen in anhydrous solvents. Column chromatography was performed with Merck silica gel 60 (70-230 mesh). Thin layer chromatography was performed on Aldrich 250 $\mu \mathrm{m}$ silica gel TLC plates or Analtech RPS-F reverse phase TLC plate and visualization was achieved with vanillin in sulfuric acid or ammonium molybdate, cerium ammonium sulfate and sulfuric acid in water, both followed by heating. 1D NMR spectra were recorded on a Bruker Avance $300 \mathrm{MHz}$, a Bruker DRX $400 \mathrm{MHz}$ or a Bruker AMX 600 MHz spectrometer and 2D NMR spectra were recorded on a Bruker AMX $600 \mathrm{MHz}$ spectrometer. NMR spectra were run in $\mathrm{CDCl}_{3}$ or $\mathrm{CD}_{3} \mathrm{OD}$ and referenced to protonated solvent peak or solvent ${ }^{13} \mathrm{C}$ NMR peak as internal standards $\left(\mathrm{CDCl}_{3}: 7.24 \mathrm{ppm}\right.$ and 77.0 ppm, $\mathrm{CD}_{3} \mathrm{OD}: 3.30 \mathrm{ppm}$ and $49.0 \mathrm{ppm}$ for ${ }^{1} \mathrm{H}$ NMR and ${ }^{13} \mathrm{C}$ NMR, respectively). When $\mathrm{D}_{2} \mathrm{O}$ was the solvent, DSS (sodium 3-(trimethylsilyl)-1-propanesulfonate) was used as an external reference for ${ }^{13} \mathrm{C}$ NMR and referenced to the residual $\mathrm{D}_{2} \mathrm{O}$ signal at $4.78 \mathrm{ppm}$ for ${ }^{1} \mathrm{H}$ NMR. As an internal standard, the peak for TMS was referenced at $0 \mathrm{ppm}$. Splitting patterns are designated as s, singlet; d, doublet; dd, doublet of doublet; ddd, doublet of 
doublet of doublet; t, triplet; m, multiplet. High-resolution mass spectrometry was performed by the University of Iowa, Department of Chemistry, High Resolution Mass Spectrometry Facility with a Micromass, Inc. Autospec mass spectrometer. Optical rotations were determined using Jasco P-1020 polarimeter. CD spectra were recorded with an On-line instrument system, Inc. CARY-17 CD spectrometer.

\section{Mycothiol (1-D-myo-inosityl 2-deoxy-2-( $N$-acetamido-L-cysteinamido)- $\alpha$-D-}

\section{glucopyranoside, MSH) and Mycothiol disulfide (MSSM): $1.58 \mathrm{~mL}$ of $7.8 \%$}

$\mathrm{Mg}(\mathrm{OMe})_{2}(1.17 \mathrm{mmole})$ in methanol was added dropwise to solution of compound $\mathbf{1 1}$ (51.7 mg, 0.06 mmole) in methanol $(4 \mathrm{~mL})$. The reaction was stirred for $2 \mathrm{~h}$ at room temperature and 1\% TFA in methanol was added until the $\mathrm{pH}$ decreased to between $6 \sim 7$. The resulting reaction mixture was purified by silica gel column chromatography (55:45 ethyl acetate: methanol) to yield $11.5 \mathrm{mg}$ of MSH (0.024 mmole, $40 \%)$ and $16.8 \mathrm{mg}$ of MSSM (0.017 mmole, 29\%) as a white powder.: $\mathrm{R}_{f}(\mathrm{MSH}) 0.65$ and $\mathrm{R}_{f}(\mathrm{MSSM}) 0.34$ (5:5:0.1 EtOAc: $\mathrm{MeOH}: \mathrm{HCOOH}) ;[\alpha]_{\mathrm{D}}^{25}(\mathrm{MSH})+43.5^{\circ}\left(\right.$ c $\left.0.1, \mathrm{H}_{2} \mathrm{O}\right)$ and $[\alpha]_{\mathrm{D}}{ }^{25}$ (MSSM) $+42^{\circ}$ (c 0.1, $\mathrm{H}_{2} \mathrm{O}$ ); CD MSH $\left(60 \mu \mathrm{M}, \mathrm{H}_{2} \mathrm{O}\right)[\Phi]_{226}-0.26$, $[\Phi]_{191}+4.4$; MSSM $\left(60 \mu \mathrm{M}, \mathrm{H}_{2} \mathrm{O}\right)[\Phi]_{270}-0.07,[\Phi]_{223}+1.9,[\Phi]_{190}+4.7 ;$ HRMS m/z calculated for $\mathrm{C}_{17} \mathrm{H}_{30} \mathrm{~N}_{2} \mathrm{O}_{12} \mathrm{SNa}\left[\mathrm{MNa}^{+}\right]$509.1417, found 509.1424 (MSH) and calculated for $\mathrm{C}_{34} \mathrm{H}_{58} \mathrm{~N}_{4} \mathrm{O}_{24} \mathrm{~S}_{2} \mathrm{Na}\left[\mathrm{MNa}^{+}\right]$993.2780, found 993.2743 (MSSM).

Mycothiol bimane (MSmB): A solution of monobromobimane (mBBr, $3.1 \mathrm{mg}$, $0.011 \mathrm{mmole}$ ) in acetonitrile ( $200 \mu \mathrm{l}$ ) was added to solution of MSH (3.7 mg, 0.008 
Scheme 1. Numbering of synthetic MSH, MSSM and MSmB

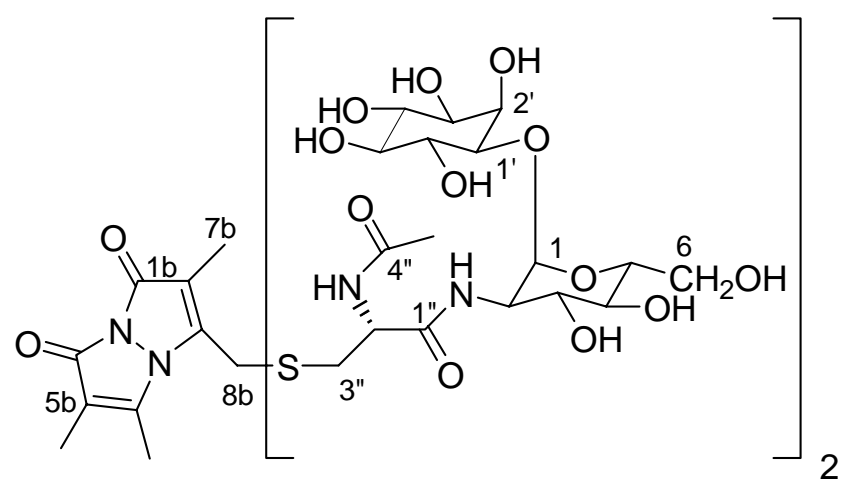

Table $1 .{ }^{1} \mathrm{H}$ and ${ }^{13} \mathrm{C}$ NMR data of synthetic MSH and MSSM.

\begin{tabular}{|c|c|c|c|c|c|}
\hline & \multicolumn{2}{|r|}{$\mathrm{MSH}$} & \multicolumn{2}{|r|}{ MSSM } \\
\hline & & $\delta_{\mathrm{C}}$ & $\delta_{\mathrm{H}}$, mult. $J(\mathrm{~Hz})$ & $\delta_{\mathrm{C}}$ & $\delta_{\mathrm{H}}$, mult. $J(\mathrm{~Hz})$ \\
\hline \multirow[t]{7}{*}{ D-glucosamine } & 1 & 102.0 & $5.13 \mathrm{~d} 3.7$ & 101.9 & $5.15 \mathrm{~d} 3.7$ \\
\hline & 2 & 56.7 & 3.98 dd $10.7,3.7$ & 56.8 & 3.98 dd $10.8,3.7$ \\
\hline & 3 & 73.6 & 3.79 dd $10.7,9.7$ & 73.5 & 3.83 dd 10.9, 9.6 \\
\hline & 4 & 72.9 & 3.47 t 9.5 & 72.9 & 3.49 t 9.6 \\
\hline & 5 & 75.3 & $3.85 \mathrm{~m}$ & 75.4 & $3.87 \mathrm{~m}$ \\
\hline & 6 & 63.4 & $3.87 \mathrm{~m}$ & 63.4 & 3.89 dd $10.1,2.3$ \\
\hline & & & $3.76 \mathrm{~m}$ & & 3.78 dd $10.1,2.3$ \\
\hline \multirow[t]{6}{*}{ D-myo-inositol } & $1^{\prime}$ & 81.8 & 3.58 dd $10.1,2.8$ & 81.7 & 3.60 dd $10.1,2.8$ \\
\hline & $2^{\prime}$ & 74.5 & 4.12 t 2.8 & 74.5 & 4.20 t 2.7 \\
\hline & $3^{\prime}$ & 73.9 & 3.51 dd $10.0,2.8$ & 73.9 & 3.53 dd $10.0,2.7$ \\
\hline & $4^{\prime}$ & 74.8 & 3.61 t 9.7 & 74.9 & 3.64 t 9.8 \\
\hline & $5^{\prime}$ & 77.0 & 3.27 t 9.4 & 77.0 & 3.30 t 9.4 \\
\hline & $6^{\prime}$ & 74.9 & 3.77 t 9.6 & 75.0 & 3.79 t 9.9 \\
\hline$N$-Acetyl- & $1 "$ & 175.0 & & 175.1 & \\
\hline \multirow[t]{5}{*}{ L-cysteine } & $2 "$ & 58.6 & 4.51 dd 6.7, 5.6 & 55.6 & 4.72 dd $9.1,4.9$ \\
\hline & $3 "$ & 28.5 & 2.93 dd 14.1, 5.4 & 41.8 & 3.26 dd $14.2,4.7$ \\
\hline & & & 2.89 dd $14.1,6.9$ & & $2.98 \mathrm{dd} 14.2,9.2$ \\
\hline & $4 "$ & 177.2 & & 177.3 & \\
\hline & $5 "$ & 24.6 & $2.06 \mathrm{~s}$ & 24.6 & $2.09 \mathrm{~s}$ \\
\hline
\end{tabular}

${ }^{a}{ }^{1} \mathrm{H}$ NMR $\left(\mathrm{D}_{2} \mathrm{O}\right)$ recorded at $600 \mathrm{MHz},{ }^{13} \mathrm{C}$ NMR $\left(\mathrm{D}_{2} \mathrm{O}\right)$ recorded at $100 \mathrm{MHz}$.

${ }^{\text {b }}{ }^{\mathrm{H}} \mathrm{NMR}\left(\mathrm{D}_{2} \mathrm{O}\right)$ recorded at $400 \mathrm{MHz},{ }^{13} \mathrm{C}$ NMR $\left(\mathrm{D}_{2} \mathrm{O}\right)$ recorded at $100 \mathrm{MHz}$. 
mmole) and $\mathrm{NaHCO}_{3}(1.6 \mathrm{mg}, 0.019 \mathrm{mmole})$ in $\mathrm{H}_{2} \mathrm{O}(2 \mathrm{~mL})$. The reaction mixture was stirred at room temperature in the dark and was monitored by reverse phase TLC. After 2 h stirring, $\mathrm{H}_{2} \mathrm{O}(8 \mathrm{~mL})$ was added and then extracted with $\mathrm{CH}_{2} \mathrm{Cl}_{2}(5 \mathrm{~mL}, \times 2)$ to remove unreacted $\mathrm{mBBr}$. The aqueous phase was lyophilized and resulting mixture was subsequently separated with Supelco LC-18 SPE tubes (95:5 $\mathrm{H}_{2} \mathrm{O}$ : acetonitrile). Column chromatography gave $4 \mathrm{mg}$ of MSmB (0.006 mmole, 78\%) as amorphous yellow powder: $\mathrm{R}_{f} 0.5$ (reverse phase, bright blue band at $366 \mathrm{~nm}, 9: 1 \mathrm{H}_{2} \mathrm{O}$ :acetonitrile); $[\alpha]_{\mathrm{D}}^{25}+26^{\circ}$ (c 0.1, $\left.\mathrm{H}_{2} \mathrm{O}\right) ; \mathrm{CD}\left(60 \mu \mathrm{M}, \mathrm{H}_{2} \mathrm{O}\right)[\Phi]_{219}-1.14,[\Phi]_{189}+6.6 ;{ }^{1} \mathrm{H}$ NMR $\left(\mathrm{D}_{2} \mathrm{O}, 400 \mathrm{MHz}\right) \delta 5.12$ (d, $J=3.7 \mathrm{~Hz}, \mathrm{H}-1$ ), 4.57 (dd, $J=8.4,5.2 \mathrm{~Hz}, \mathrm{H}-2$ ”), 4.18 (t, $J=2.8 \mathrm{~Hz}, \mathrm{H}-2$ '), 3.96 (dd, $J=10.8,3.7$ Hz, H-2), 3.95 (d, $J=14.9$ Hz, H8b-a), 3.9 (d, $J=14.9$ Hz, H8b-b), 3.88 (m, H-6a), 3.87 (m, H-5), 3.81 (m, H-6b), 3.79 (t, J=10.4 Hz, H-3), 3.76 (t, J= 9.7 Hz, H-6'), 3.63 (t, $J=9.8 \mathrm{~Hz}, \mathrm{H}-4$ '), 3.58 (dd, $J=10.1,2.9 \mathrm{~Hz}, \mathrm{H}-1$ '), 3.52 (dd, $J=10.2,2.8 \mathrm{~Hz}, \mathrm{H}-$ 3’), 3.48 (t, $J=9.9$ Hz, H-4), 3.29 (t, $J=9.4$ Hz, H-5’), 3.14 (dd, $J=14.0,5.2 \mathrm{~Hz}, \mathrm{H}-3$ ”a), 2.96 (dd, J= 14.0, 8.5 Hz, H-3”b), 2.46 (s, H-9b), 2.04 (s, H-5”), 1.87 (s, H-7b), 1.80 (s, H-10b); ${ }^{13} \mathrm{C}$ NMR* ( $\left.\mathrm{D}_{2} \mathrm{O}, 100 \mathrm{MHz}\right) \delta 177.0$ (C-4”), 174.7 (C-1”), 166.1 (C-6b), 165.3 (C-1b), 152.7 (C-4b), 151.0 (C-3b), 116.5 (C-2b), 114.4 (C-5b), 101.9 (C-1), 81.9 (C-1'), 77.0 (C-5’), 75.3 (C-5), 74.9 (C-6’), 74.87 (C-4’), 74.5 (C-2’), 73.9 (C-3’), 73.5 (C-3), 72.9 (C-4), 63.4 (C-6), 56.7 (C-2), 55.7 (C-2”), 36.2 (C-3”), 27.7 (C-8b), 24.5 (C-5”), 13.9 (C-9b), 8.9 (C-7b), 8.4 (C-10b); HRMS m/z calculated for $\mathrm{C}_{27} \mathrm{H}_{40} \mathrm{~N}_{4} \mathrm{O}_{14} \mathrm{SNa}\left[\mathrm{MNa}^{+}\right]$ 699.2159, found 699.2157.

* Chemical shift differences in our data versus the literature ${ }^{1}$ for MSmB are due to our use of DSS as an external reference versus $d_{6}$-DMSO used as an internal reference standard. NMR spectra for synthetic MSmB were identical to that for MSmB isolated from Nocardia. 
Reduction of MSSM to MSH: A sample of $7.3 \mathrm{mg}$ of bis(2-mercaptoethyl)sulfone (BMS, 0.039 mmole) in $1 \mathrm{~mL}$ of $\mathrm{H}_{2} \mathrm{O}$ was added to $5.4 \mathrm{mg}$ of MSSM (0.006 mmole) in $1 \mathrm{~mL}$ of $\mathrm{H}_{2} \mathrm{O}$. The resulting solution was stirred for 5 days at room temperature, followed by the addition $9 \mathrm{~mL}$ of $\mathrm{H}_{2} \mathrm{O}$. The reaction mixture was extracted with ethyl acetate $(10 \mathrm{~mL}, \times 3)$ to remove unreacted BMS and oxidized BMS, the byproduct of the reaction. Lyophilization of the aqueous phase gave $5.4 \mathrm{mg}$ of MSH (0.11 mmole, 100\%). All spectral data were identical to those for synthetic MSH from 11. All $\mathrm{H}_{2} \mathrm{O}$ was refluxed with argon for $1 \mathrm{~h}$ before use.

Compound 8: A mixture of 1:2 of $\alpha / \beta$ trichloroactimidate (7, $820 \mathrm{mg}, 1.72$ mmole, scheme 3), ${ }^{2}$ D-2,3,4,5,6-penta-O-acetyl-myo-inositol (6, 807 mg, 2.07 mmole, Scheme 2), ${ }^{3-5}$ in $\mathrm{CH}_{2} \mathrm{Cl}_{2}(12 \mathrm{~mL})$ containing $4 \AA$ molecular sieves was stirred for $30 \mathrm{~min}$ at room temperature and then cooled to $0{ }^{\circ} \mathrm{C}$. A $2 \mathrm{~N}$ solution of trifluoromethanesulfonate (TMSOTf, $0.172 \mathrm{mmole}$ ) in $\mathrm{CH}_{2} \mathrm{Cl}_{2}$ was added and the solution was stirred at $0{ }^{\circ} \mathrm{C}$ for $1 \mathrm{~h}$. $\mathrm{NaHCO}_{3}$ (550 mg, 7.14 mmole) was added to the reaction that was then filtered with Celite and concentrated. Column chromatography $\left(7: 2 \mathrm{CHCl}_{3}: \mathrm{Et}_{2} \mathrm{O}\right)$ gave 8 (674 mg, 0.96 mmole, $56 \%$ ) as a white powder.; $\mathrm{R}_{f} 0.25$ (3:1, $\mathrm{CHCl}_{3}: \mathrm{Et}_{2} \mathrm{O}$ ); $[\alpha]_{\mathrm{D}}{ }^{25}+74^{\circ}$ (c 0.1, $\left.\mathrm{CHCl}_{3}\right) ;{ }^{1} \mathrm{H} \mathrm{NMR}\left(\mathrm{CDCl}_{3}+\mathrm{TMS}, 400 \mathrm{MHz}\right) \delta 5.62(\mathrm{t}, J=2.8 \mathrm{~Hz}, \mathrm{H}-2$ '), 5.59 (t, $J=10.1$ Hz, H-6'), 5.51 (t, $J=10.3$ Hz, H-4'), 5.29 (dd, $J=10.7,10.8$ Hz, H-3), 5.13 (d, $J=3.4$ Hz, H-1), 5.11 (t, $J=9.8 \mathrm{~Hz}, \mathrm{H}-5$ '), 5.01 (dd, $J=10.6,2.9 \mathrm{~Hz}, \mathrm{H}-3$ '), 4.99 (t, $J=10.2 \mathrm{~Hz}, \mathrm{H}-4$ ), 4.25 (dd, $J=12.4,4.9$ Hz, H-6a), 4.09 (m, H-5), 4.08 (dd, $J=12.4,2.0$ Hz, H-6b), 4.00 (dd, $J=10.2,2.8 \mathrm{~Hz}, \mathrm{H}-1$ '), 3.27 (dd, $J=10.7,3.8 \mathrm{~Hz}, \mathrm{H}-2$ ), 2.26 (s, $\mathrm{C}_{3} \mathrm{C}=\mathrm{O}$ ), 2.11 (s, $\mathrm{C}_{3} \mathrm{C}=\mathrm{O}$ ), 2.07 (s, $\underline{\mathrm{C}}_{3} \mathrm{C}=\mathrm{O}$ ), 2.06 (s, $\underline{\mathrm{C}}_{3} \mathrm{C}=\mathrm{O}$ ), 2.04 (s, $\left.\underline{\mathrm{C}}_{3} \mathrm{C}=\mathrm{O}\right), 2.02\left(\mathrm{~s}, \mathrm{C}_{3} \mathrm{C}=\mathrm{O}\right.$ ), 
$2.01\left(\mathrm{~s}, \mathrm{CH}_{3} \mathrm{C}=\mathrm{O} \times 2\right) ;{ }^{13} \mathrm{C}-\mathrm{NMR}\left(\mathrm{CDCl}_{3}+\mathrm{TMS}, 100 \mathrm{MHz}\right) \delta 170.7\left(\mathrm{CH}_{3} \underline{\mathrm{C}}=\mathrm{O}\right), 170.3$ $\left(\mathrm{CH}_{3} \underline{\mathrm{C}}=\mathrm{O}\right), 170.0\left(\mathrm{CH}_{3} \underline{\mathrm{C}}=\mathrm{O}\right), 169.8\left(\mathrm{CH}_{3} \underline{\mathrm{C}}=\mathrm{O}\right), 169.68\left(\mathrm{CH}_{3} \underline{\mathrm{C}}=\mathrm{O}\right), 169.67\left(\mathrm{CH}_{3} \underline{\mathrm{C}}=\mathrm{O}\right)$, $169.5\left(\mathrm{CH}_{3} \underline{\mathrm{C}}=\mathrm{O}\right), 169.3\left(\mathrm{CH}_{3} \underline{\mathrm{C}}=\mathrm{O}\right), 99.8$ (C-1), 74.2 (C-1'), 71.4 (C-6'), 71.0 (C-5'), 69.5 (C-3), 69.22 (C-2’), 69.16 (C-4’), 68.84 (C-3’), 68.76 (C-5), 68.1 (C-4), 61.7 (C-6), 60.7 (C-2), $20.8\left(\underline{\mathrm{CH}}_{3} \mathrm{C}=\mathrm{O}\right), 20.62\left(\underline{\mathrm{CH}}_{3} \mathrm{C}=\mathrm{O} \times 3\right), 20.55\left(\underline{\mathrm{CH}}_{3} \mathrm{C}=\mathrm{O}\right), 20.52\left(\underline{\mathrm{CH}}_{3} \mathrm{C}=\mathrm{O}\right)$, $20.5\left(\mathrm{CH}_{3} \mathrm{C}=\mathrm{O}\right), 20.48\left(\mathrm{CH}_{3} \mathrm{C}=\mathrm{O}\right)$; HRMS m/z calculated for $\mathrm{C}_{28} \mathrm{H}_{37} \mathrm{~N}_{3} \mathrm{O}_{18} \mathrm{Na}\left[\mathrm{MNa}^{+}\right]$ 726.1970, found 726.1987 .

Compound 9: Compound 8 (282 mg, 0.4 mmole) was added to a suspension of $150 \mathrm{mg}$ of $5 \%$ palladium on carbon in $12 \mathrm{~mL}$ of ethyl acetate, followed by the addition of $1 \mathrm{~N} \mathrm{HCl}(480 \mu \mathrm{l}, 0.48$ mmole). The reaction mixture was stirred under hydrogen (14.7 psi) for $6 \mathrm{~h}$ at room temperature. The reaction solution was filtered with Celite and concentrated under vacuum to give compound 9 (230 mg, $0.32 \mathrm{mmole}, 81 \%$ ) as a white powder.; $[\alpha]_{\mathrm{D}}{ }^{25}+37^{\circ}$ (c 0.2, MeOH); ${ }^{1} \mathrm{H} \mathrm{NMR}\left(\mathrm{CD}_{3} \mathrm{OD}, 300 \mathrm{MHz}\right) \delta 5.72$ (t, $J=2.7 \mathrm{~Hz}$, H-2'), 5.49 (t, $J=9.9$ Hz, H-6'), 5.46 (t, $J=9.6$ H-4'), 5.25 (t, $J=9.6$ Hz, H-3), 5.27 (d, $J=$ 3.3 Hz, H-1), 5.23 (m, H-5'), 5.20 (m, H-3’), 5.00 (t, J=9.9 Hz, H-4), 4.46 (dd, J= 9.9, 2.7 Hz, H-1'), 4.25 (dd, J= 12.6, 4.5 Hz, H-6a), 4.11 (m, H-5), 4.08 (m, H-6b), 3.64 (dd, $J=10.8,3.0 \mathrm{~Hz}, \mathrm{H}-2), 2.27$ (s, $\underline{\mathrm{C}}_{3} \mathrm{C}=\mathrm{O}$ ), 2.084 (s, $\underline{\mathrm{C}}_{3} \mathrm{C}=\mathrm{O}$ ), 2.078 (s, $\underline{\mathrm{C}}_{3} \mathrm{C}=\mathrm{O}$ ), 2.07 (s, $\mathrm{C}_{3} \mathrm{C}=\mathrm{O}$ ), 2.02 (s, $\underline{\mathrm{H}}_{3} \mathrm{C}=\mathrm{O}$ ), 2.01 (s, $\underline{\mathrm{C}}_{3} \mathrm{C}=\mathrm{O}$ ), 1.98 (s, $\left.\underline{\mathrm{C}}_{3} \mathrm{C}=\mathrm{O}\right), 1.97$ (s, $\underline{\mathrm{C}}_{3} \mathrm{C}=\mathrm{O}$ );

${ }^{13} \mathrm{C}$ NMR $\left(\mathrm{CD}_{3} \mathrm{OD}, 75 \mathrm{MHz}\right) \delta 172.5\left(\mathrm{CH}_{3} \underline{\mathrm{C}}=\mathrm{O}\right), 172.3\left(\mathrm{CH}_{3} \underline{\mathrm{C}}=\mathrm{O}\right), 172.2\left(\mathrm{CH}_{3} \underline{\mathrm{C}}=\mathrm{O}\right)$, $171.9\left(\mathrm{CH}_{3} \underline{\mathrm{C}}=\mathrm{O}\right), 171.5\left(\mathrm{CH}_{3} \underline{\mathrm{C}}=\mathrm{O}\right), 171.47\left(\mathrm{CH}_{3} \underline{\mathrm{C}}=\mathrm{O}\right), 171.4\left(\mathrm{CH}_{3} \underline{\mathrm{C}}=\mathrm{O} \times 2\right), 99.7(\mathrm{C}-1)$, 76.1 (C-1'), 73.2 (C-6’), 73.0 (C-5’), 71.4 (C-3), 70.64 (C-2'), 70.56 (C-4'), 70.52 (C-3’), 70.45 (C-5), 69.7 (C-4), 62.7 (C-6), 53.7 (C-2), $20.9\left(\underline{\mathrm{CH}}_{3} \mathrm{C}=\mathrm{O}\right), 20.83\left(\mathrm{CH}_{3} \mathrm{C}=\mathrm{O} \times 2\right)$, 
$20.77\left(\underline{\mathrm{C}} \mathrm{H}_{3} \mathrm{C}=\mathrm{O}\right), 20.7\left(\underline{\mathrm{CH}}_{3} \mathrm{C}=\mathrm{O}\right), 20.6\left(\underline{\mathrm{C}} \mathrm{H}_{3} \mathrm{C}=\mathrm{O} \times 2\right), 20.58\left(\underline{\mathrm{CH}}_{3} \mathrm{C}=\mathrm{O}\right) ; \mathrm{HRMS} \mathrm{m} / \mathrm{z}$ calculated for $\mathrm{C}_{28} \mathrm{H}_{40} \mathrm{NO}_{18} \mathrm{Na}\left[\mathrm{MNa}^{+}\right]$678.2245, found 678.2282.

N,S-diacetyl-L-cysteine (10): L-Cysteine hydrochloride (9 g, 57.1 mmole) was added to $\mathrm{NaOH}$ ( $6.4 \mathrm{~g}, 160 \mathrm{mmole}$ ) in $40 \mathrm{~mL}$ of $\mathrm{H}_{2} \mathrm{O}$ at $0{ }^{\circ} \mathrm{C}$, followed by the addition of acetic anhydride (102 mmole). The reaction mixture was stirred for 30 min at $0{ }^{\circ} \mathrm{C}$, then adjusted to $\mathrm{pH} 2$ with $6 \mathrm{~N} \mathrm{HCl}$. The mixture was extracted with ethyl acetate $(40 \mathrm{~mL}, \times 4)$. The organic phase was combined, dried $\left(\mathrm{Na}_{2} \mathrm{SO}_{4}\right)$ and concentrated under vacuum to yield $9.8 \mathrm{~g}$ of $N, S$-diacetyl-L-cysteine ( $47.8 \mathrm{mmole}, 84 \%$ ) as a white powder: $\mathrm{R}_{f} 0.42$ (90:5:1 CHCl 3 :EtOAc: $\left.\mathrm{CH}_{3} \mathrm{COOH}\right) ;[\alpha]_{\mathrm{D}}{ }^{25}-26^{\circ}$ (c 0.67, MeOH); ${ }^{1} \mathrm{H}$ NMR (DMSO- $d_{6}+$ TMS, $400 \mathrm{MHz}$ ) $\delta 12.89$ (s, OH), 8.26 (d, $J=8.2 \mathrm{~Hz}, \mathrm{NH}$ ), 4.34 (ddd, $J=8.3,8.3,5.0 \mathrm{~Hz}$, H-2”), 3.34 (dd, $J=13.6,5.0$ Hz, H-3”a), 3.02 (dd, $J=13.6,8.4$ Hz, H-3”b), 2.33 (s, Sacetyl $\mathrm{CH}_{3}$ ), 1.83 (s, H-5”); ${ }^{13} \mathrm{C}$ NMR (DMSO- $d_{6}+$ TMS, $100 \mathrm{MHz}$ ) $\delta 194.5$ (S-acetyl $\underline{\mathrm{C}}=\mathrm{O}), 171.6$ (C-1”), 169.2 (C-4”), 51.3 (C-2”), 30.3 (S-acetyl CH 3 ), 30.0 (C-3”), 22.2 (C5”); HRMS $m / z$ calculated for $\mathrm{C}_{7} \mathrm{H}_{11} \mathrm{NO}_{4} \mathrm{SNa}\left[\mathrm{MNa}^{+}\right]$228.0306, found 228.0284 .

Compound 11: To a stirred solution of 9 (202 mg, $0.28 \mathrm{mmole}$ ) and 10 (69.6 mg, 0.34 mmole) in $7 \mathrm{~mL}$ DMF, 2,4,6-collidine (0.57 mmole) was added. The mixture was stirred for $15 \mathrm{~min}$ at $0{ }^{\circ} \mathrm{C}$ before solid 1-hydroxy-7-azabenzotriazole (HOAt, $46 \mathrm{mg}, 0.34$ mmole) and $O$-(7-azabenzotriazol-1-yl)- $N, N, N^{\prime}, N^{\prime}$-tetramethyluronium hexafluorophosphate (HATU, 129 mg, 0.34 mmole) were added. The reaction mixture was stirred for $2 \mathrm{~h}$ at $0{ }^{\circ} \mathrm{C}$, warmed to room temperature, and stirred for an additional $22 \mathrm{~h} . \mathrm{H}_{2} \mathrm{O}$ (50 $\mathrm{mL}$ ) was added and the mixture extracted three times with $40 \mathrm{~mL}$ of ethyl acetate. The 
combined organic extracts were washed twice with $40 \mathrm{~mL}$ of $1 \mathrm{~N} \mathrm{HCl}$, twice with $40 \mathrm{~mL}$ of saturated $\mathrm{NaHCO}_{3}$ and twice with $40 \mathrm{~mL}$ of saturated $\mathrm{NaCl}$. The organic extract was dried $\left(\mathrm{Na}_{2} \mathrm{SO}_{4}\right)$ and concentrated under vacuum. Purification by column chromatography (8:2 hexane:ethyl acetate) afforded 11 (61.5 $\mathrm{mg}, 0.07 \mathrm{mmole}, 25 \%$ ) as a white powder.;

$\mathrm{R}_{f} 0.45$ (100\% EtOAc); $[\alpha]_{\mathrm{D}}{ }^{25}+47^{\circ}$ (c 0.3, MeOH); ${ }^{1} \mathrm{H}$ NMR $\left(\mathrm{CD}_{3} \mathrm{OD}, 400 \mathrm{MHz}\right) \delta 5.67$ (t, $J=2.8 \mathrm{~Hz}, \mathrm{H}-2^{\prime}$ ), 5.49 (t, $J=9.8 \mathrm{~Hz}, \mathrm{H}-6^{\prime}$ ), 5.45 (t, $\left.J=10.0 \mathrm{~Hz}, \mathrm{H}-4^{\prime}\right), 5.27$ (t, $J=9.8$ Hz, H-5’), 5.24 (dd, $J=9.9,2.8$ Hz, H-3'), 5.11 (dd, $J=10.9,9.9$ Hz, H-3), 5.06 (d, $J=3.8$ Hz, H-1), 4.99 (t, J= 9.9 Hz, H-4), 4.57 (dd, J= 7.9, 4.4 Hz, H-2”), 4.40 (dd, $J=10.0,3.0$ Hz, H-1'), 4.22 (m, H-6a), 4.18 (dd, J= 10.9, 3.8 Hz, H-2), 4.13 (m, H-5), 4.11 (m, H-6b), 3.38 (dd, J= 14.0, 4.4 Hz, H-3”a), 2.99 (dd, J=14.0, 7.9 Hz, H-3”b), 2.35 (s, S-acetyl $\mathrm{C}_{3}$ ), 2.29 (s, $\underline{\mathrm{C}}_{3} \mathrm{C}=\mathrm{O}$ ), 2.10 (s, $\underline{\mathrm{C}}_{3} \mathrm{C}=\mathrm{O}$ ), 2.03 (s, C-5”), 2.00 (s, $\underline{\mathrm{C}}_{3} \mathrm{C}=\mathrm{O}$ ), 1.997 (s, $\mathrm{C}_{3} \mathrm{C}=\mathrm{O}$ ), 1.987 (s, $\left.\underline{\mathrm{C}}_{3} \mathrm{C}=\mathrm{O}\right), 1.984$ (s, $\left.\underline{\mathrm{C}}_{3} \mathrm{C}=\mathrm{O}\right) ; 1.962$ (s, $\left.\underline{\mathrm{C}}_{3} \mathrm{C}=\mathrm{O}\right), 1.96$ (s, $\left.\mathrm{C}_{3} \mathrm{C}=\mathrm{O}\right) ;{ }^{13} \mathrm{C}$ NMR (CD $\left.{ }_{3} \mathrm{OD}, 100 \mathrm{MHz}\right) \delta 196.8$ (S-acetyl $\left.\underline{\mathrm{C}}=\mathrm{O}\right), 173.3$ (C-4"), 172.8 (C-1”), $172.4\left(\mathrm{CH}_{3} \underline{\mathrm{C}}=\mathrm{O}\right), 172.2\left(\mathrm{CH}_{3} \underline{\mathrm{C}}=\mathrm{O}\right), 172.1\left(\mathrm{CH}_{3} \underline{\mathrm{C}}=\mathrm{O}\right), 172.0\left(\mathrm{CH}_{3} \underline{\mathrm{C}}=\mathrm{O}\right), 171.53$ $\left(\mathrm{CH}_{3} \underline{\underline{C}}=\mathrm{O}\right), 171.51\left(\mathrm{CH}_{3} \underline{\mathrm{C}}=\mathrm{O}\right), 171.4\left(\mathrm{CH}_{3} \underline{\mathrm{C}}=\mathrm{O}\right), 171.3\left(\mathrm{CH}_{3} \underline{\mathrm{C}}=\mathrm{O}\right), 99.6(\mathrm{C}-1), 73.8(\mathrm{C}-$ 6'), 73.4 (C-1'), 72.6 (C-5’), 71.6 (C-3), 71.2 (C-2’), 70.8 (C-4'), 70.40 (C-3’), 70.29 (C5), 70.03 (C-4), 63.5 (C-6), 54.1 (C-2”), 54.0 (C-2), 32.7 (C-3”), 30.6 (S-acetyl $\underline{\mathrm{CH}}_{3}$ ), 22.7 (C-5”), $21.0\left(\underline{\mathrm{CH}}_{3} \mathrm{C}=\mathrm{O}\right), 20.9\left(\underline{\mathrm{CH}}_{3} \mathrm{C}=\mathrm{O}\right), 20.8\left(\underline{\mathrm{CH}}_{3} \mathrm{C}=\mathrm{O} \times 2\right), 20.7\left(\underline{\mathrm{C}} \mathrm{H}_{3} \mathrm{C}=\mathrm{O}\right), 20.6$ $\left(\underline{\mathrm{CH}_{3} \mathrm{C}}=\mathrm{O} \times 2\right), 20.5\left(\underline{\mathrm{C}} \mathrm{H}_{3} \mathrm{C}=\mathrm{O}\right)$; HRMS $\mathrm{m} / \mathrm{z}$ calculated for $\mathrm{C}_{35} \mathrm{H}_{48} \mathrm{~N}_{2} \mathrm{O}_{21} \mathrm{SNa}\left[\mathrm{MNa}^{+}\right]$ 887.2368, found 887.2364. 
Scheme 2. Synthesis of D-2,3,4,5,6-penta-O-acetyl-myo-inositol a
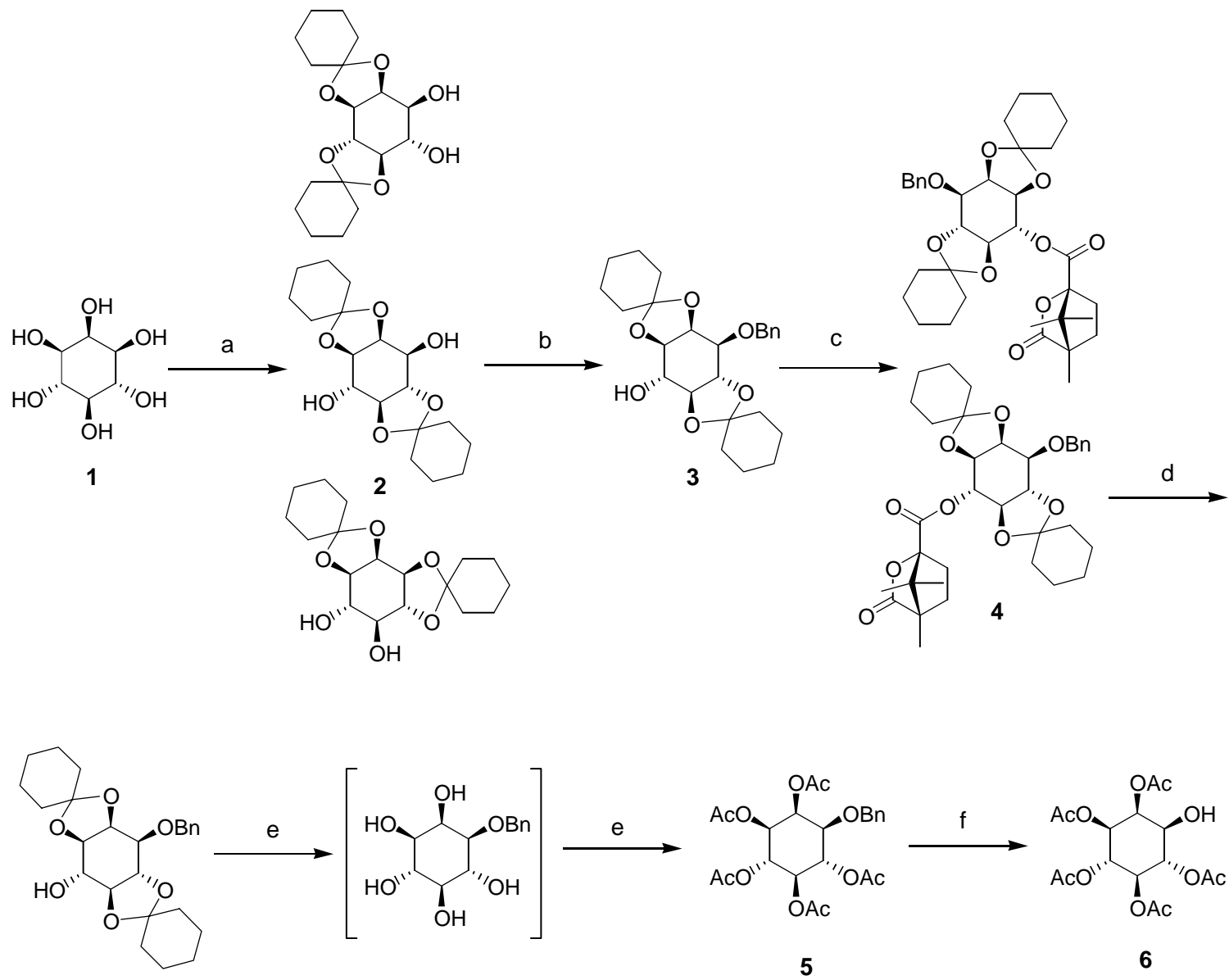

${ }^{\mathrm{a}}$ Reagents and conditions: (a) 1-ethoxycyclohexene, $p$ - $\mathrm{TsOH}, \mathrm{CH}_{2} \mathrm{Cl}_{2}, 100{ }^{\circ} \mathrm{C}, 2 \mathrm{~h}, 23 \%$; (b) $\mathrm{BnBr}, \mathrm{NaH}, \mathrm{PhMe}, 130{ }^{\circ} \mathrm{C}, 6 \mathrm{~h}$ then rt, 12 h, 54\%; (c) (1S)-(-)-camphanic chloride, DMAP, $(\mathrm{Et})_{3} \mathrm{~N}, \mathrm{CH}_{2} \mathrm{Cl}_{2}$, rt, 24 h, 49\%; (d) $\mathrm{KOH}, \mathrm{EtOH}, \mathrm{rt}, 15$ h, 97\%; (e) $\mathrm{CH}_{3} \mathrm{COOH}, \mathrm{H}_{2} \mathrm{O}, 100{ }^{\circ} \mathrm{C}, 2$ h; then $\mathrm{Ac}_{2} \mathrm{O}$, pyridine, rt, 17 h, 89\%; (f) $\mathrm{H}_{2}$, Pd-C, EtOAc, rt, 48 h, $97 \%$. 
Scheme 3. Synthesis of O-(3,4,6-tri-O-acetyl)-2-azido-2-deoxy- $\alpha, \beta$-D-glucopyranosyl) trichloroacetimidate. ${ }^{a}$

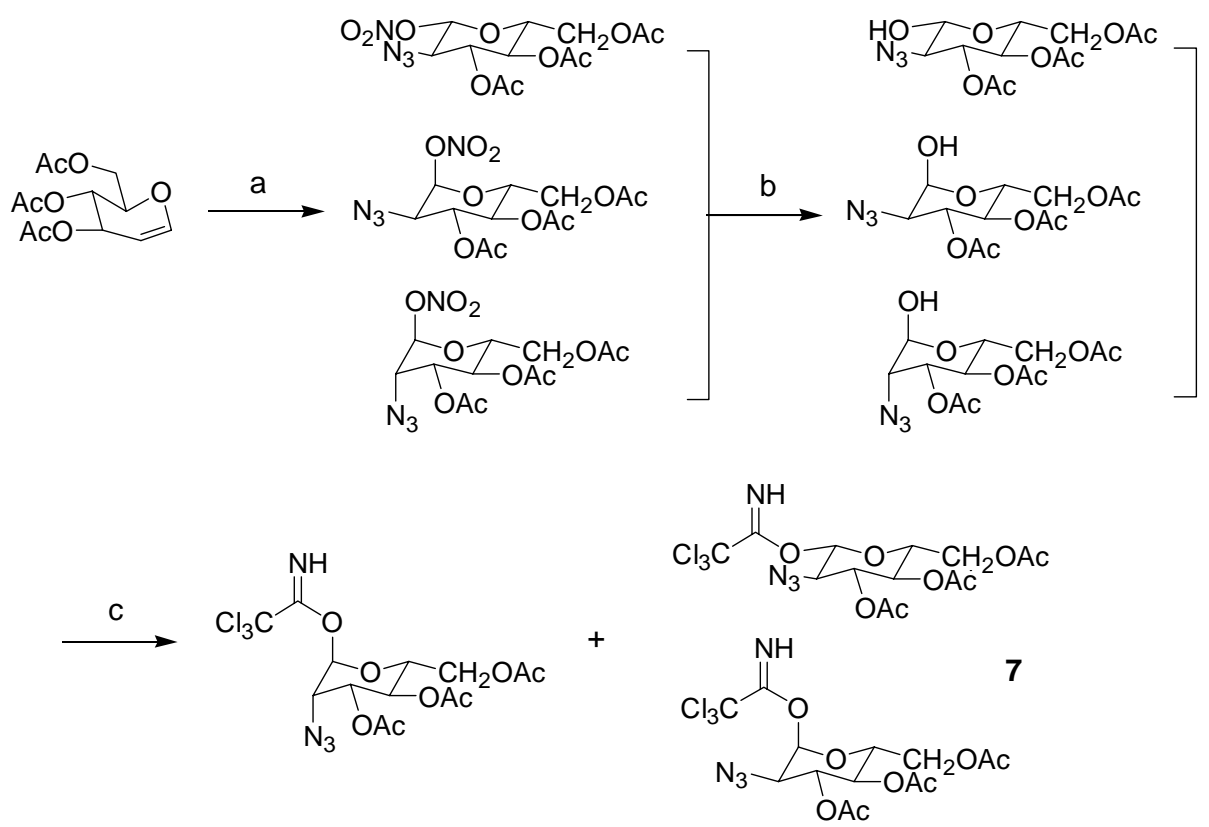

${ }^{a}$ Reagents and conditions: (a) $\mathrm{NaN}_{3}$, Ammonium cerium (IV) nitrate, $\mathrm{CH}_{3} \mathrm{CN},-15{ }^{\circ} \mathrm{C}, 12$ h, 48\%; (b) $\mathrm{NaNO}_{2}, \mathrm{H}_{2} \mathrm{O}$, dioxane, $80^{\circ} \mathrm{C}, 6$ h, 46\%; (c) $\mathrm{CCl}_{3} \mathrm{CN}, \mathrm{K}_{2} \mathrm{CO}_{3}, \mathrm{CH}_{2} \mathrm{Cl}_{2}$, rt, 6 h, $59 \%$.

\section{References}

[1] G. M. Nicholas, P.Kovac, C. A. Bewley, J. Am. Chem. Soc. 2002, 124, 3492-3493

[2] G. Grundler, R. R. Schmidt, Liebigs Ann. Chem. 1984, 11, 1826-1847.

[3] a) J. P. Vacca, S. J. Desolms, J. R. Huff, D. C. Billington, R. Baker, J. J.

Kulagowski, I. M. Mawer, Tetrahedron. 1989, 45, 5679-5702. b) J. P. Vacca, Tetrahedron. 1991, 47, 907.

[4] D. W. Gammon, D. J. Steenkamp, Bioorg. Med. Chem. 2002, 10, 875-881.

[5] a) S. Cottaz, J. S. Brimacombe, M. A. Ferguson, J. J. Chem. Soc. Perkin. Trans. 1. 1993, 23, 2945-2951. b) R. Aneja, S. G. Aneha, A. Parra, Tetrahedron-Asymmetr. 1995, 6, 17-18. 


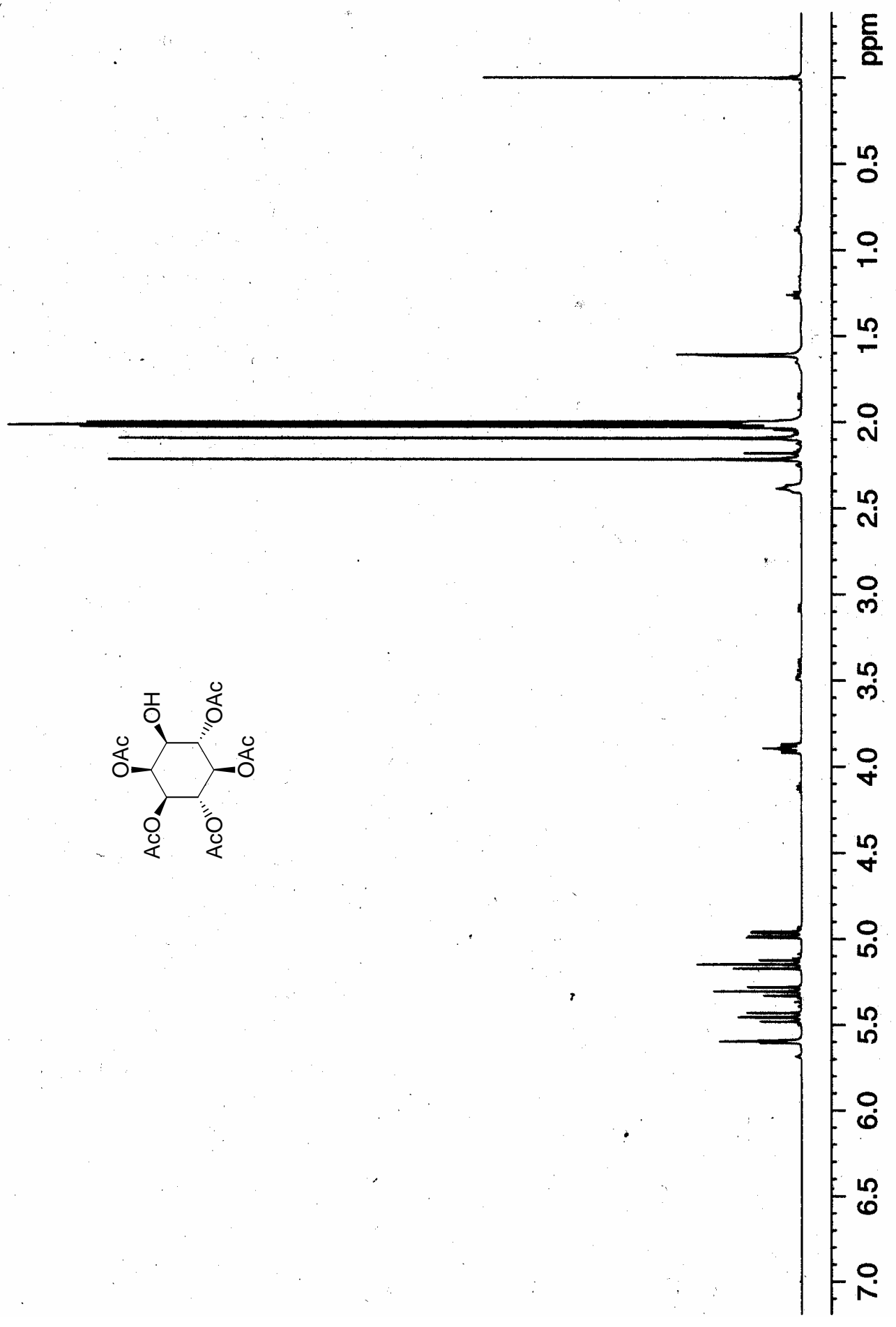

$400 \mathrm{MHz}{ }^{1} \mathrm{H}$ NMR spectrum $\left(\mathrm{CDCl}_{3}+\mathrm{TMS}\right)$ of compound 6 


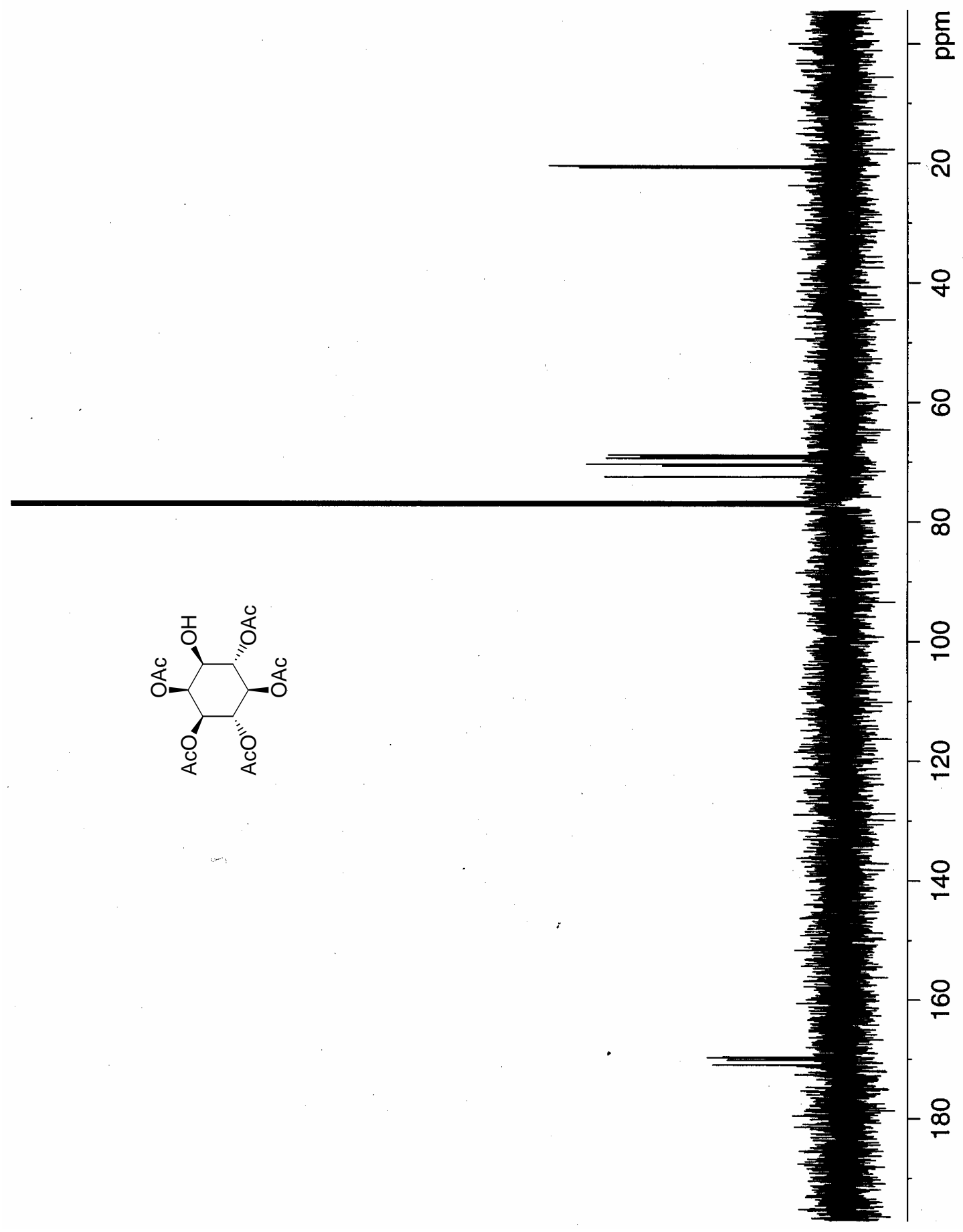

$100 \mathrm{MHz}{ }^{13} \mathrm{C}$ NMR spectrum $\left(\mathrm{CDCl}_{3}\right)$ of compound 6 


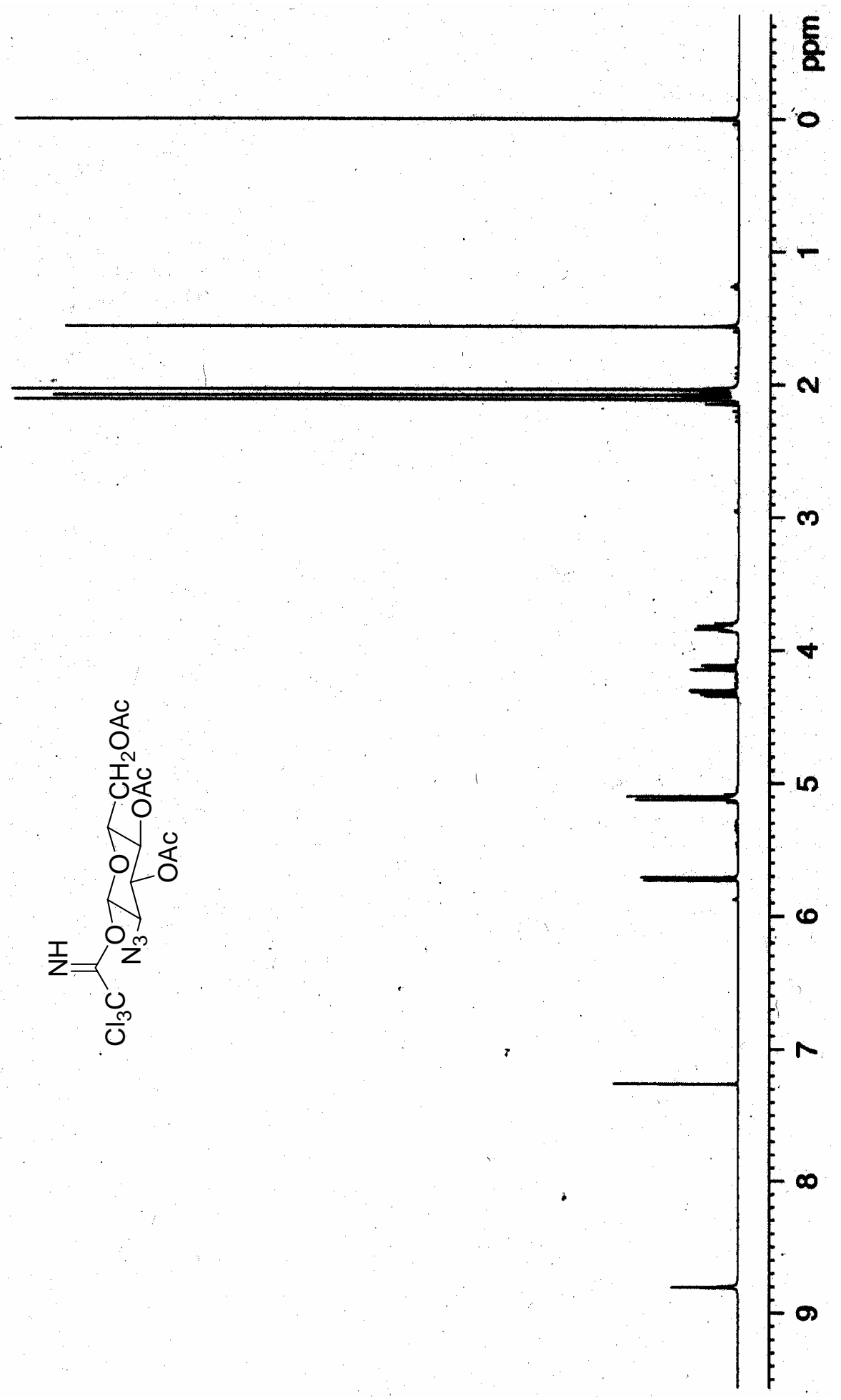

$400 \mathrm{MHz}{ }^{1} \mathrm{H}$ NMR spectrum $\left(\mathrm{CDCl}_{3}+\mathrm{TMS}\right)$ of compound 7 ( $\beta$-anomer) 


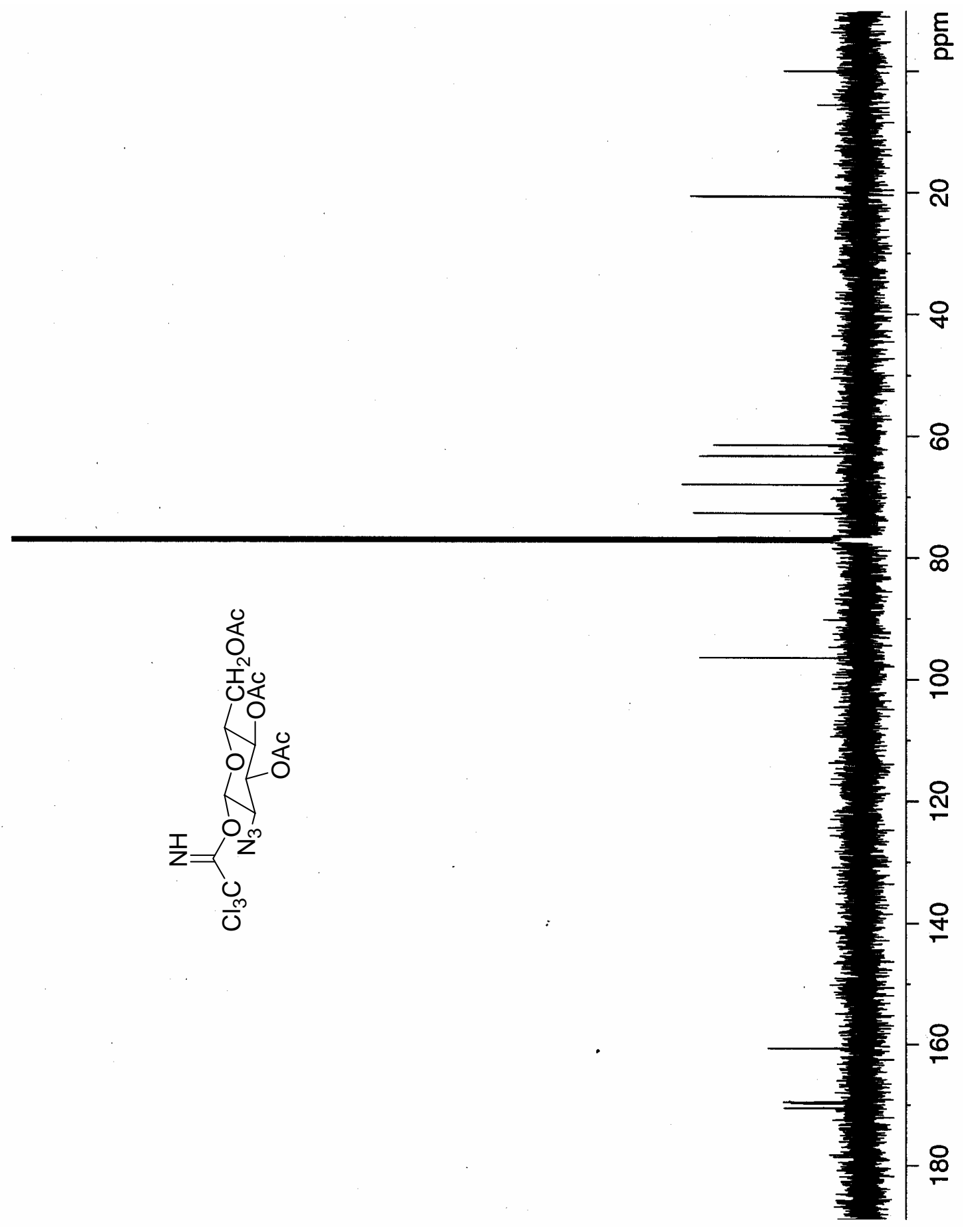

$100 \mathrm{MHz}{ }^{13} \mathrm{C}$ NMR spectrum ( $\mathrm{CDCl}_{3}+\mathrm{TMS}$ ) of compound 7 ( $\beta$-anomer) 


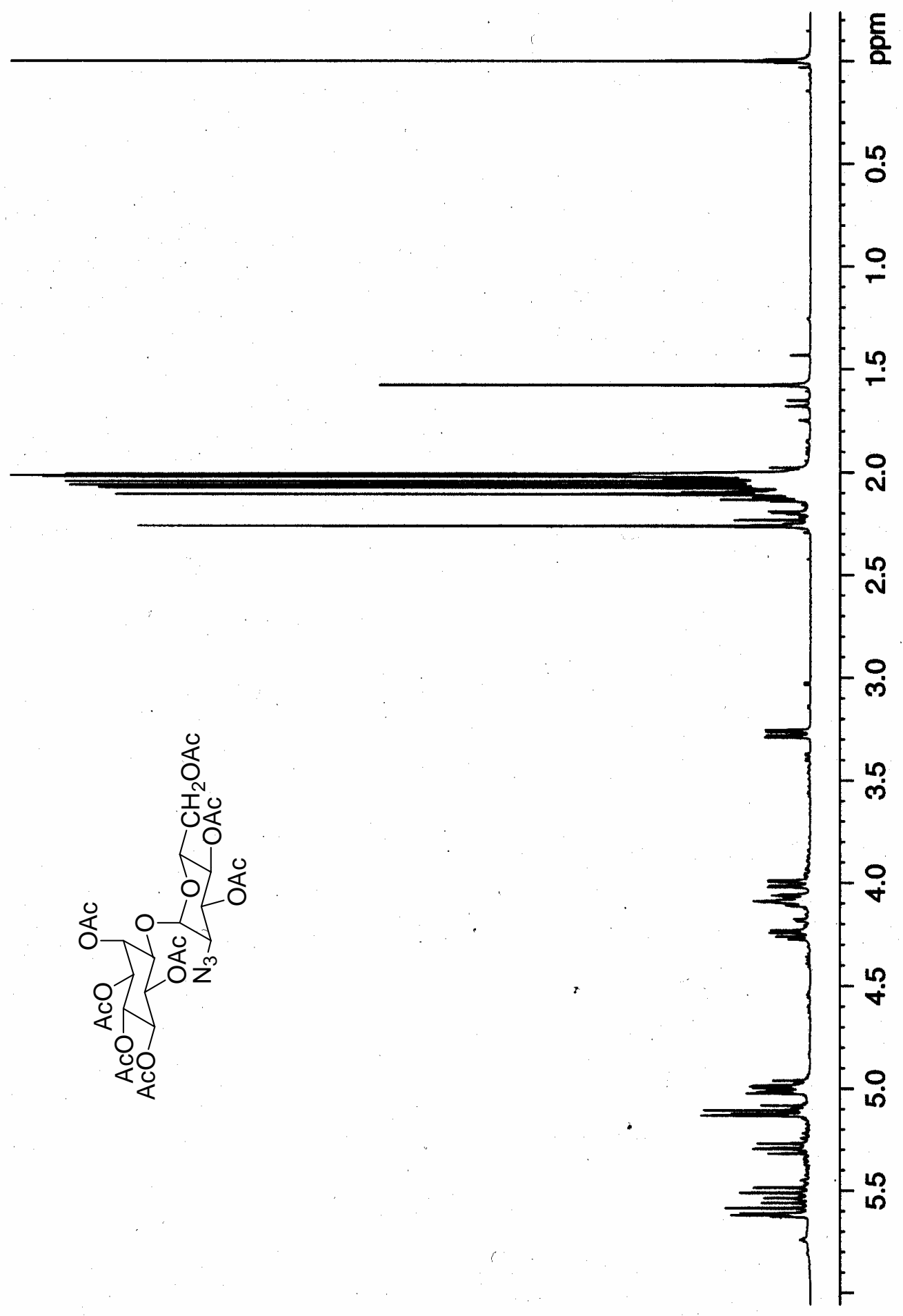

$400 \mathrm{MHz}{ }^{1} \mathrm{H}$ NMR spectrum $\left(\mathrm{CDCl}_{3}+\mathrm{TMS}\right)$ of compound 8 


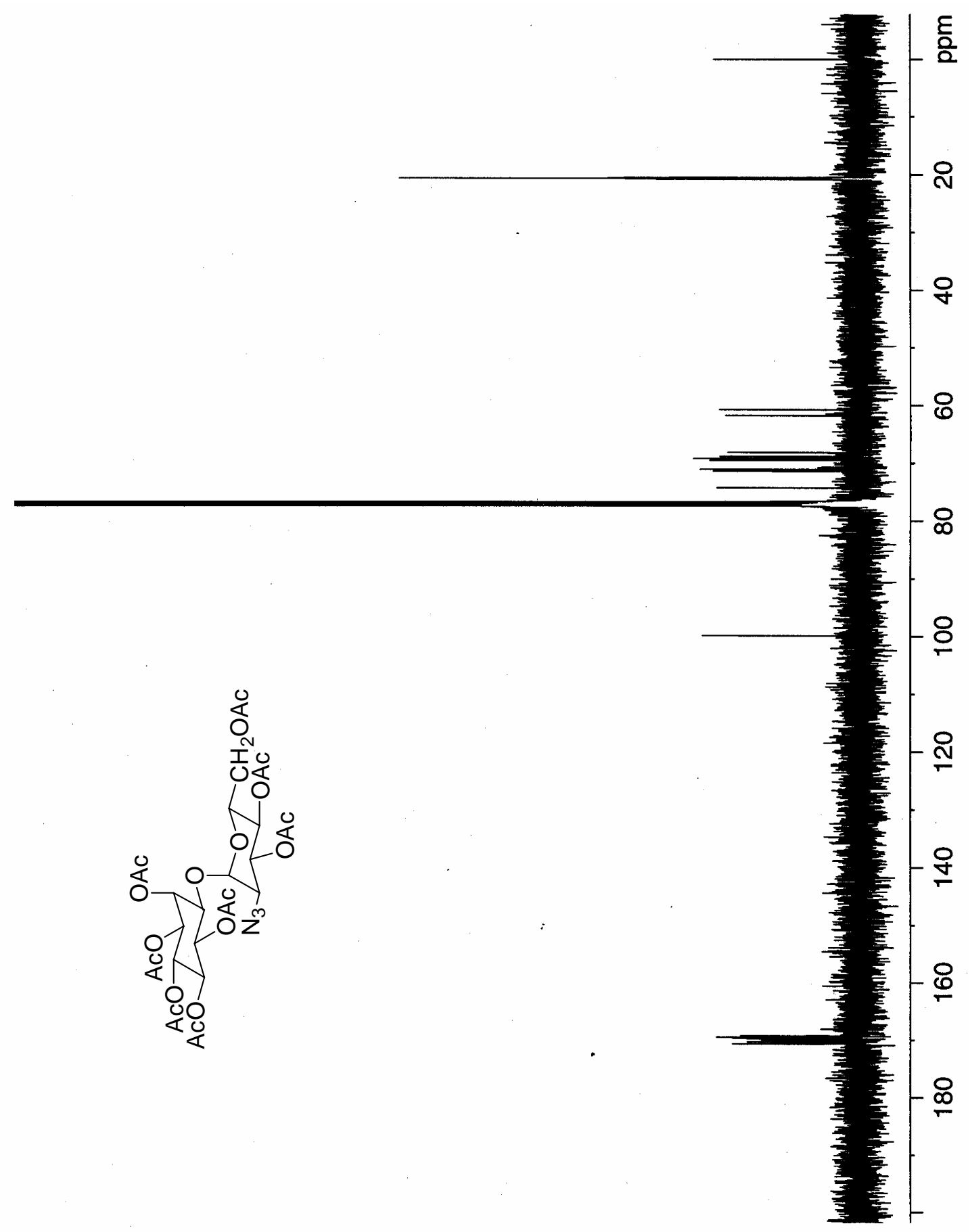

$100 \mathrm{MHz}{ }^{13} \mathrm{C}$ NMR spectrum (CDCl $3+$ TMS) of compound 8 


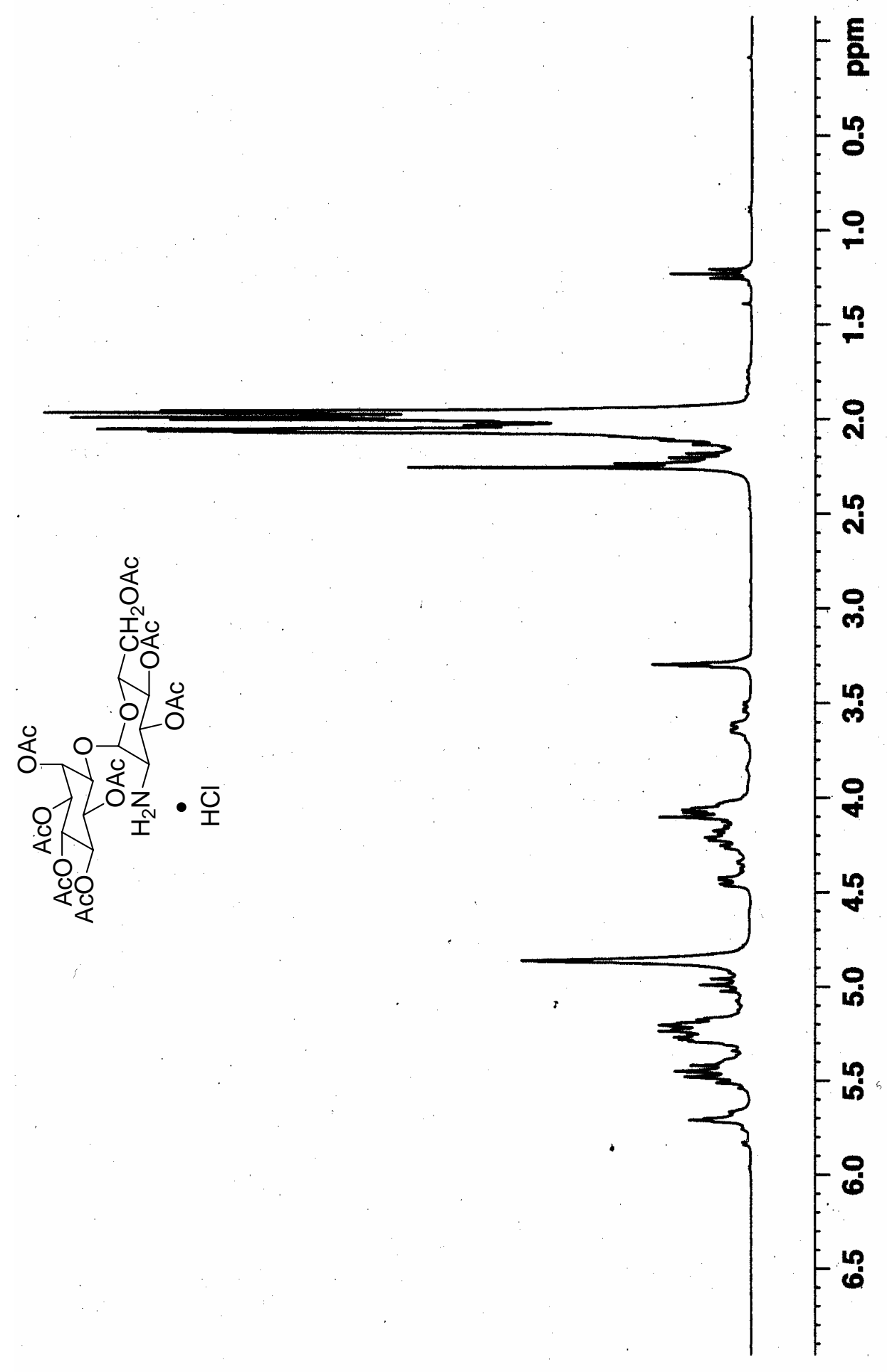

$300 \mathrm{MHz}{ }^{1} \mathrm{H}$ NMR spectrum $\left(\mathrm{CD}_{3} \mathrm{OD}\right)$ of compound 9 


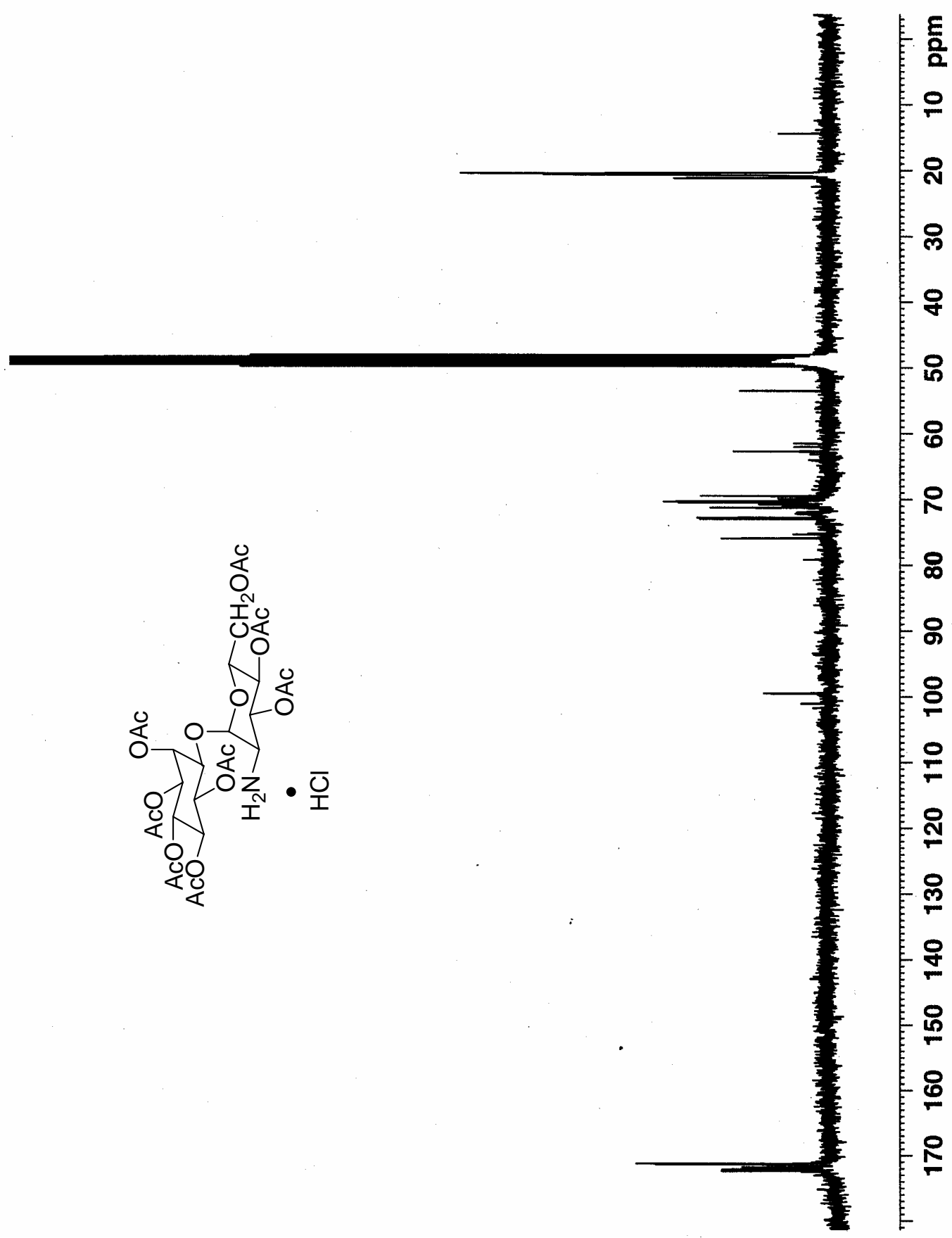

$75 \mathrm{MHz}{ }^{13} \mathrm{C}$ NMR spectrum $\left(\mathrm{CD}_{3} \mathrm{OD}\right)$ of compound 9 


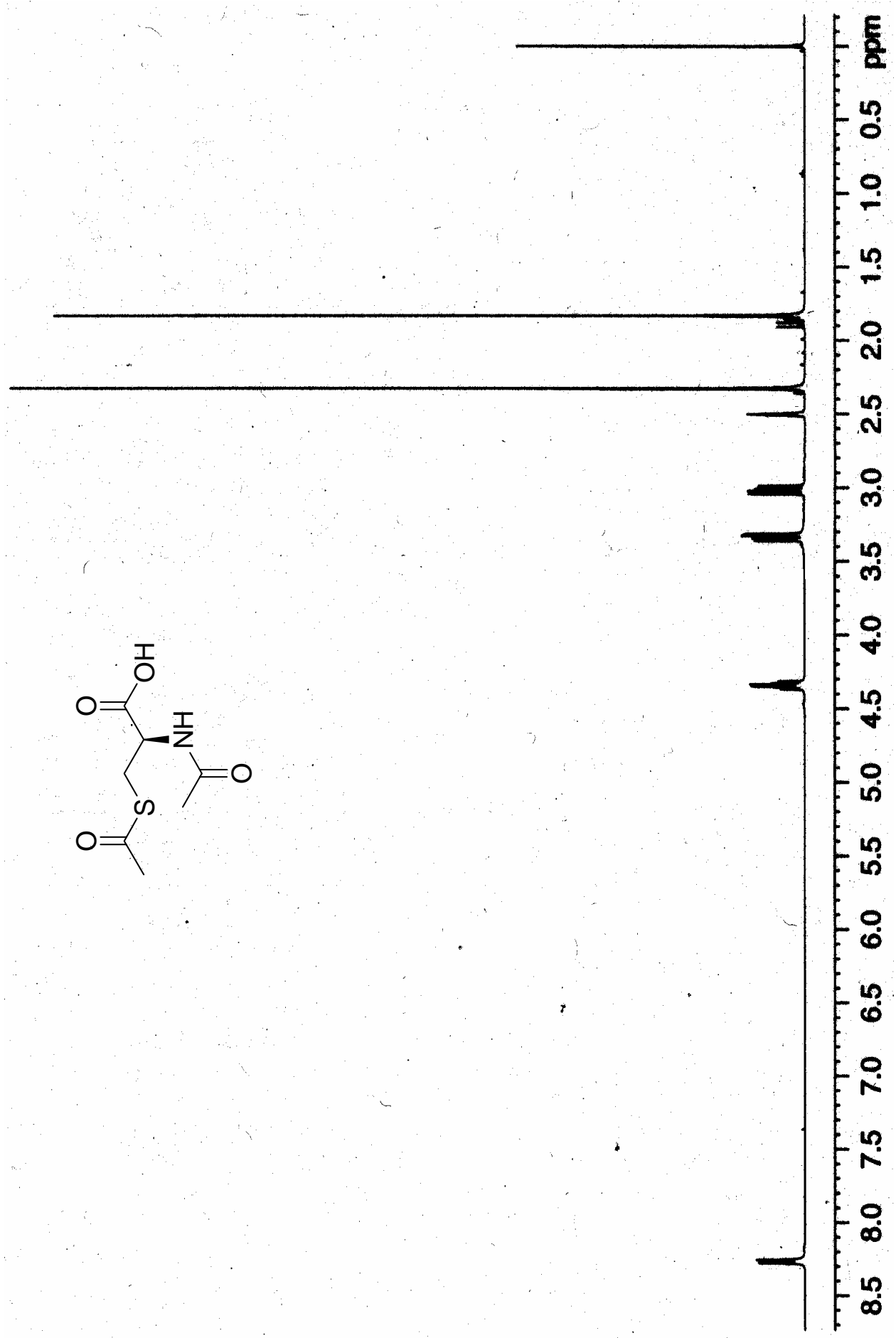

$400 \mathrm{MHz}{ }^{1} \mathrm{H}$ NMR spectrum (DMSO- $d_{6}+$ TMS) of $N$, S-diacetyl-L-cysteine (10) 


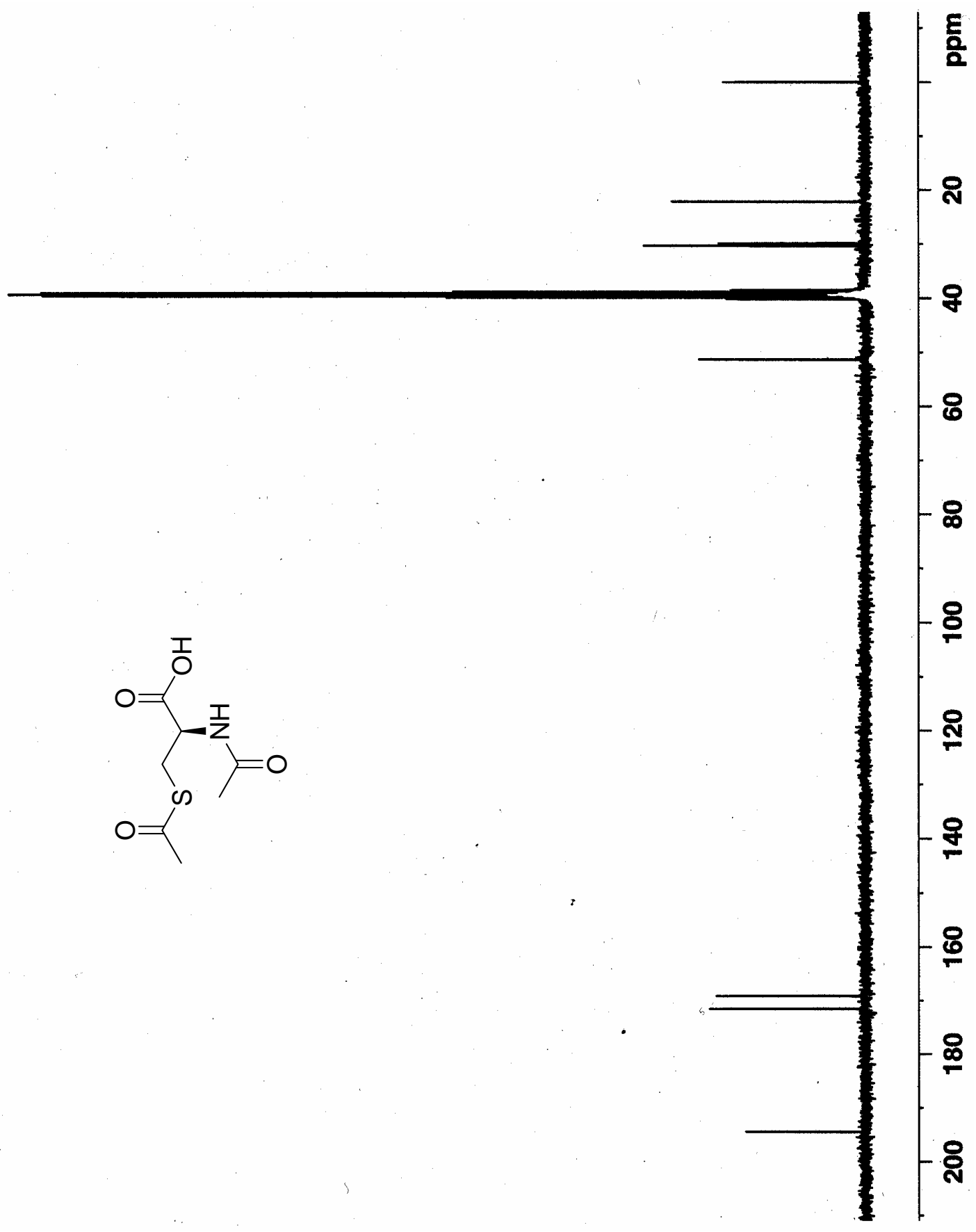

$100 \mathrm{MHz}{ }^{13} \mathrm{C}$ NMR spectrum (DMSO- $d_{6}+$ TMS) of $N$, S-diacetyl-L-cysteine (10) 


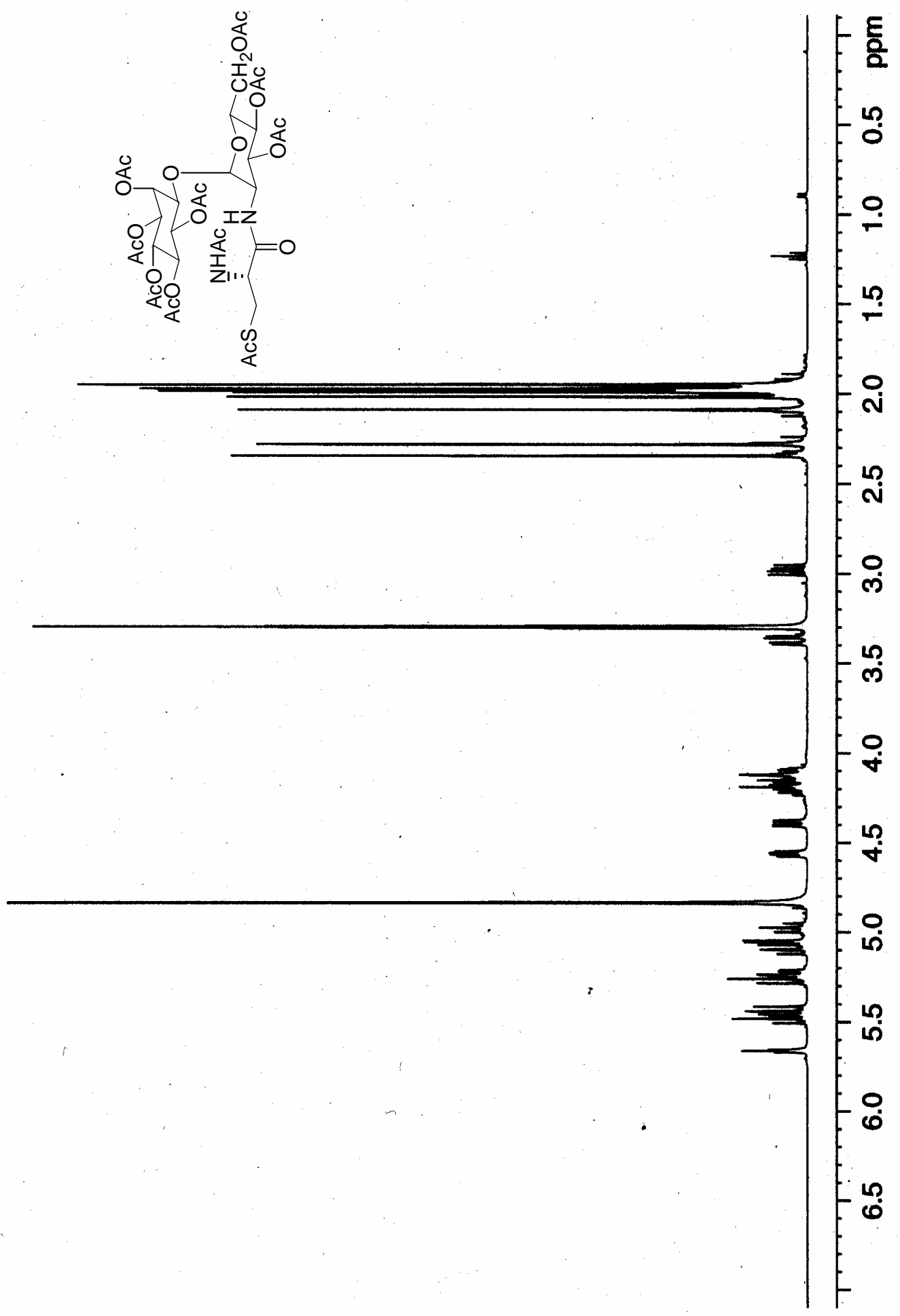

$400 \mathrm{MHz}{ }^{1} \mathrm{H}$ NMR spectrum $\left(\mathrm{CD}_{3} \mathrm{OD}\right)$ of compound 11 


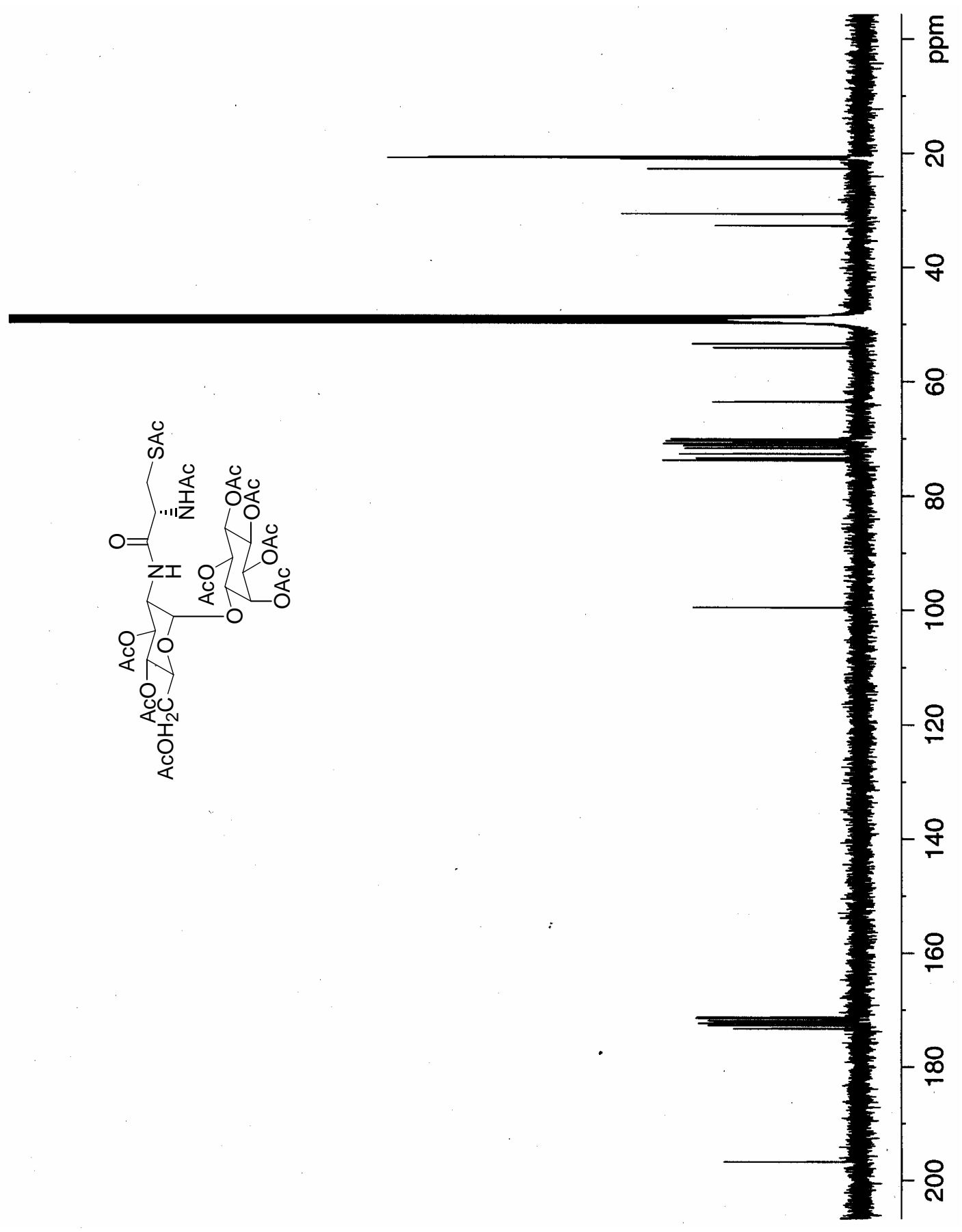

$100 \mathrm{MHz}{ }^{13} \mathrm{C}$ NMR spectrum $\left(\mathrm{CD}_{3} \mathrm{OD}\right)$ of compound 11 


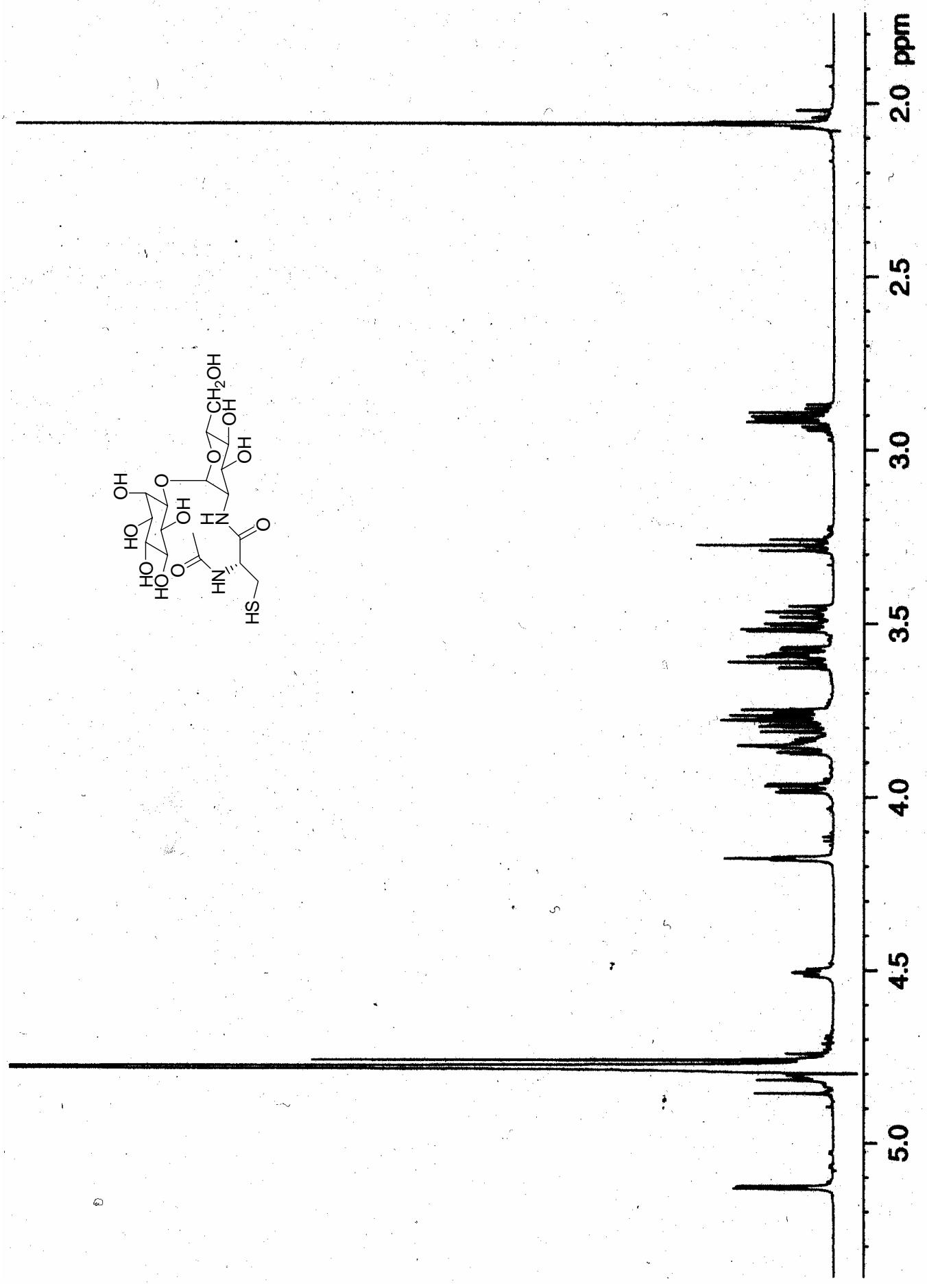

$600 \mathrm{MHz}{ }^{1} \mathrm{H}$ NMR spectrum $\left(\mathrm{D}_{2} \mathrm{O}\right)$ of $\mathrm{MSH}$ 


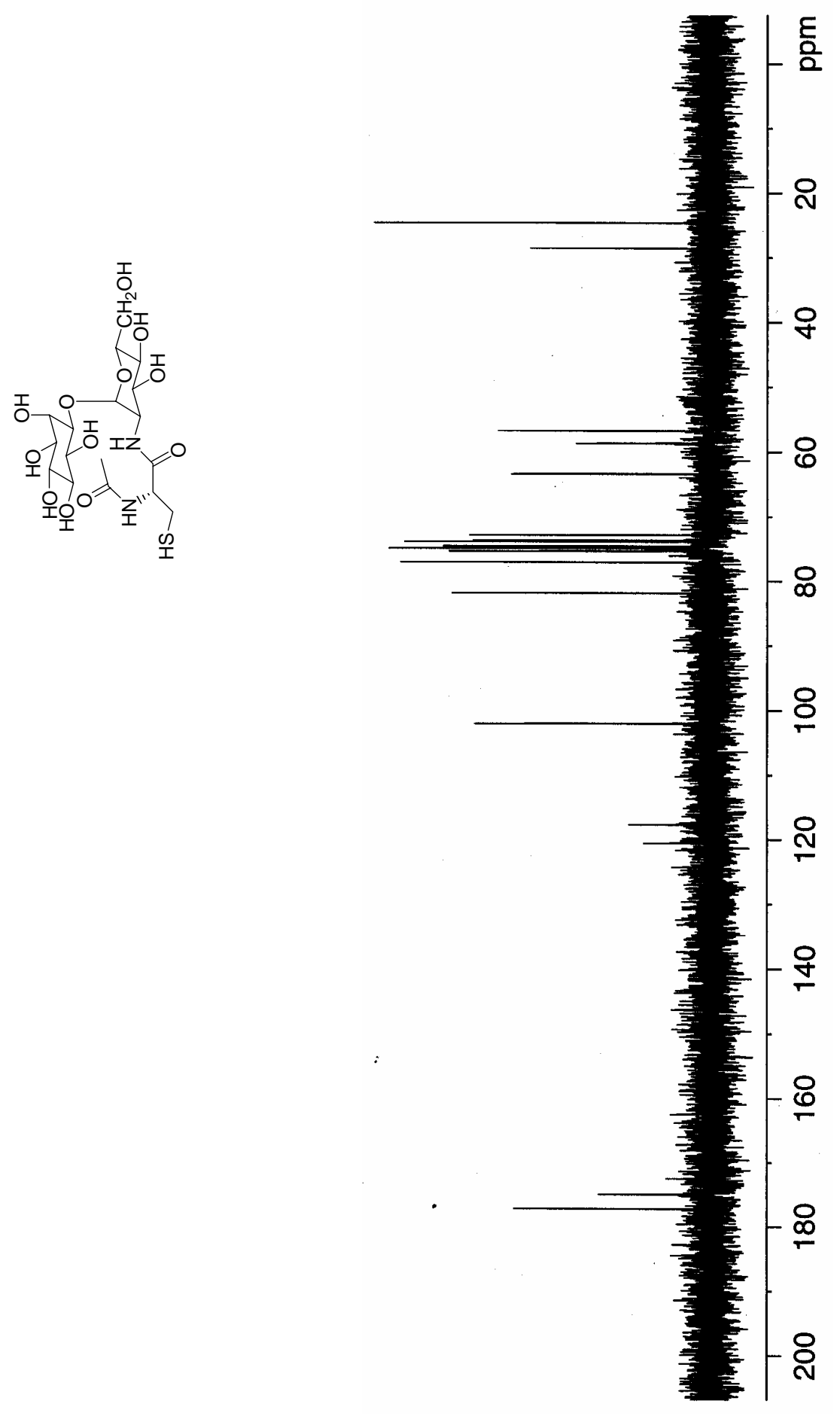

$100 \mathrm{MHz}{ }^{13} \mathrm{C}$ NMR spectrum $\left(\mathrm{D}_{2} \mathrm{O}\right)$ of $\mathrm{MSH}$ 


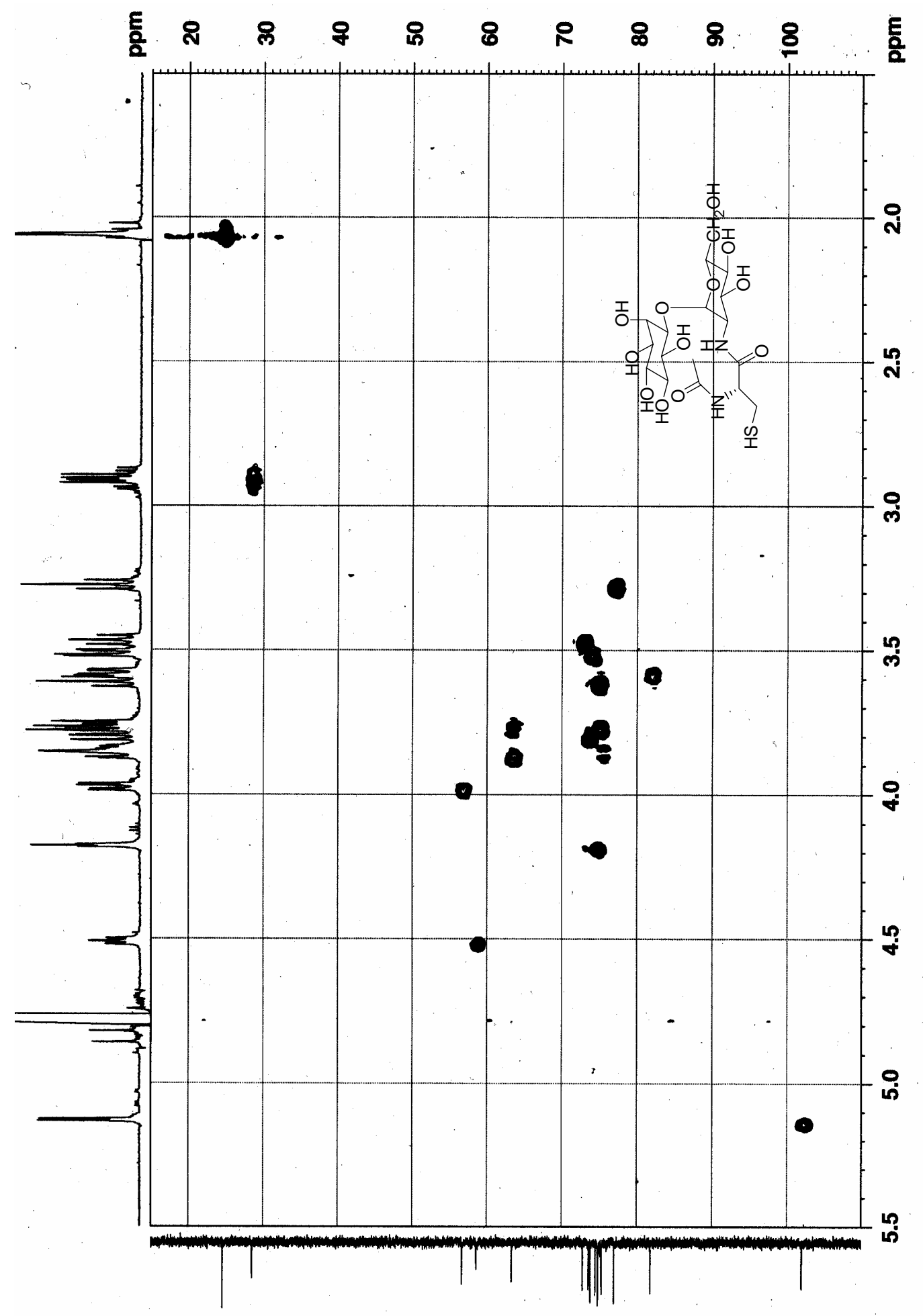

$600 \mathrm{MHz}$ NMR HMQC spectrum $\left(\mathrm{D}_{2} \mathrm{O}\right)$ of $\mathrm{MSH}$ 


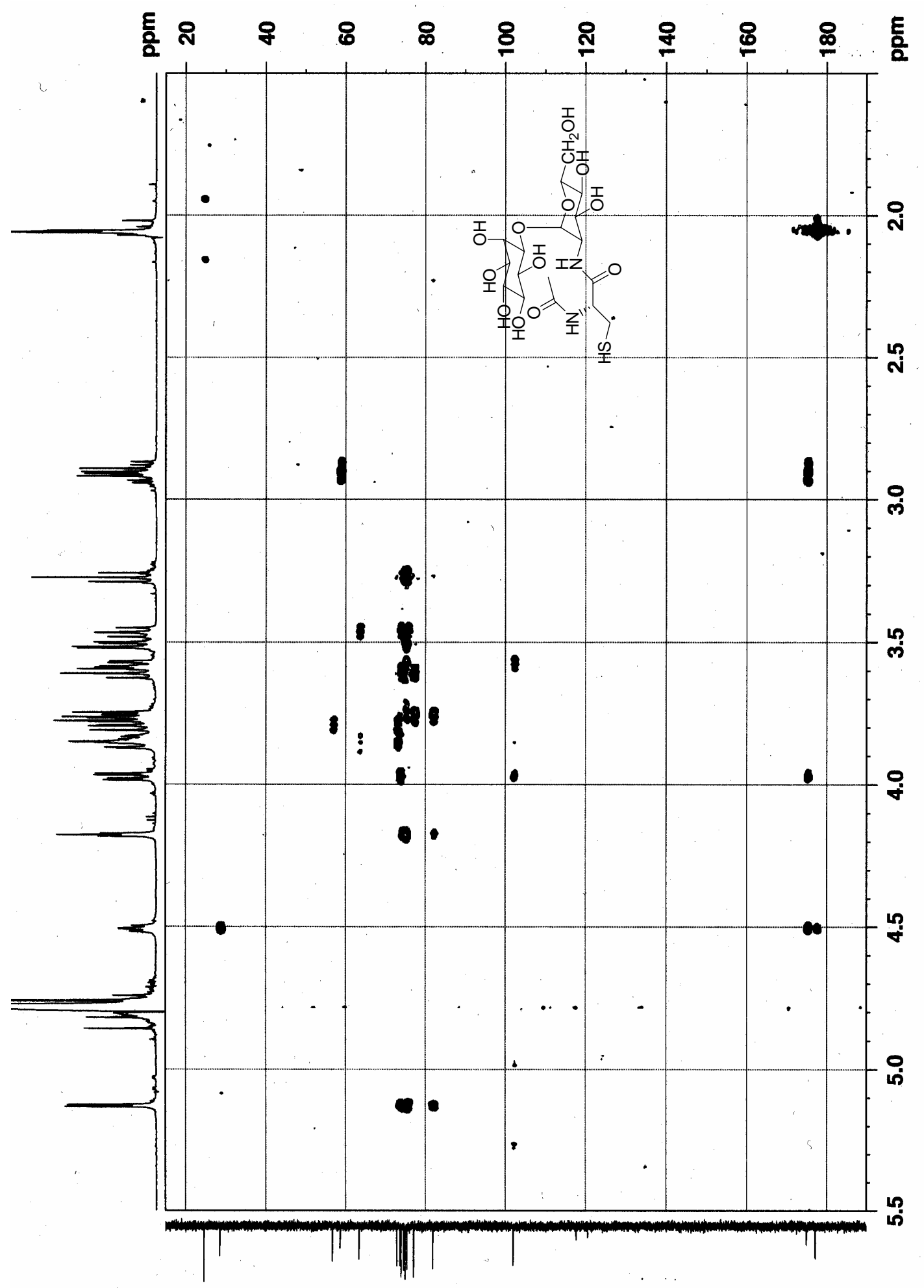

$600 \mathrm{MHz}$ NMR HMBC spectrum $\left(\mathrm{D}_{2} \mathrm{O}\right)$ of $\mathrm{MSH}$ 


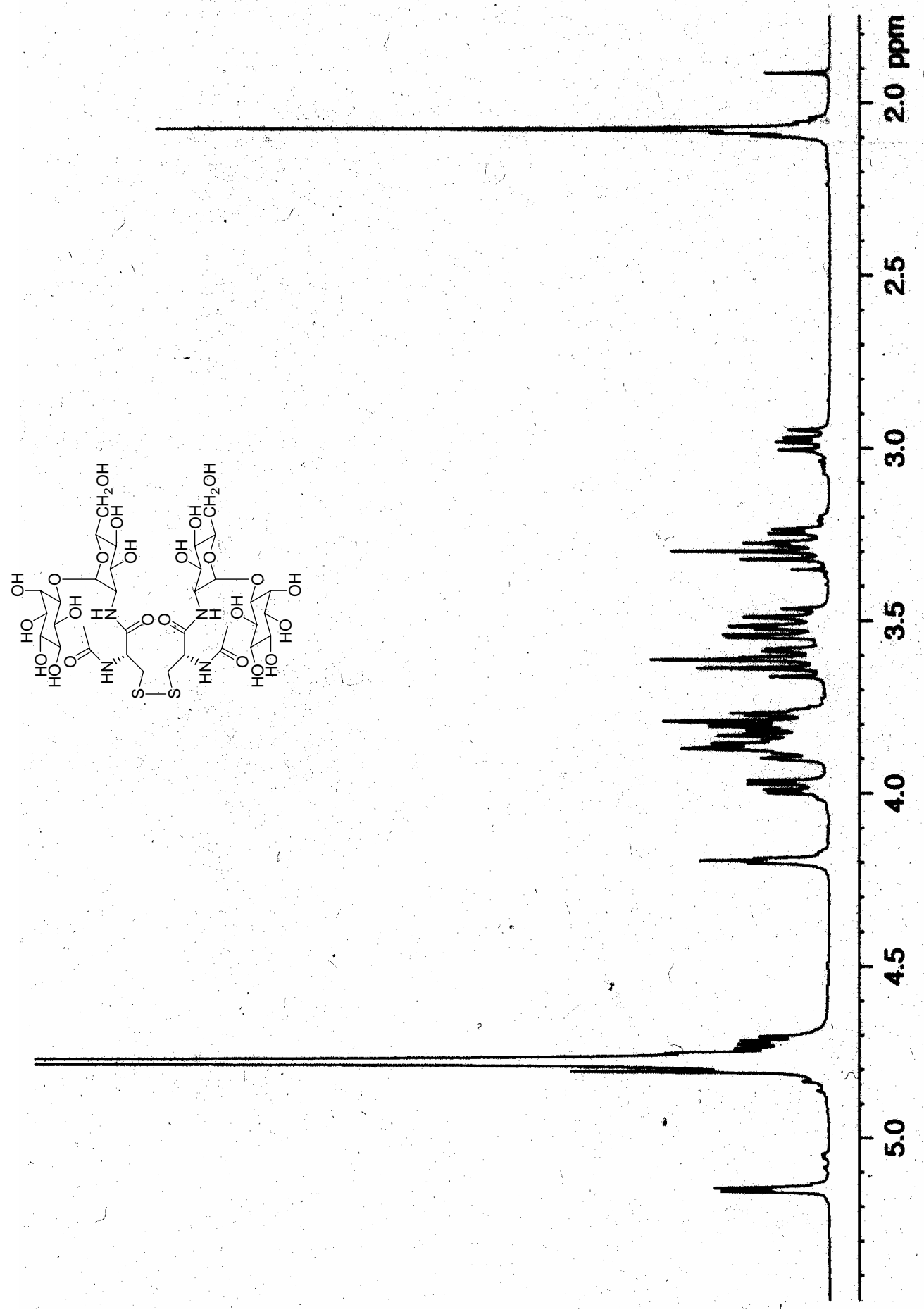

$400 \mathrm{MHz}{ }^{1} \mathrm{H}$ NMR spectrum $\left(\mathrm{D}_{2} \mathrm{O}\right)$ of MSSM 


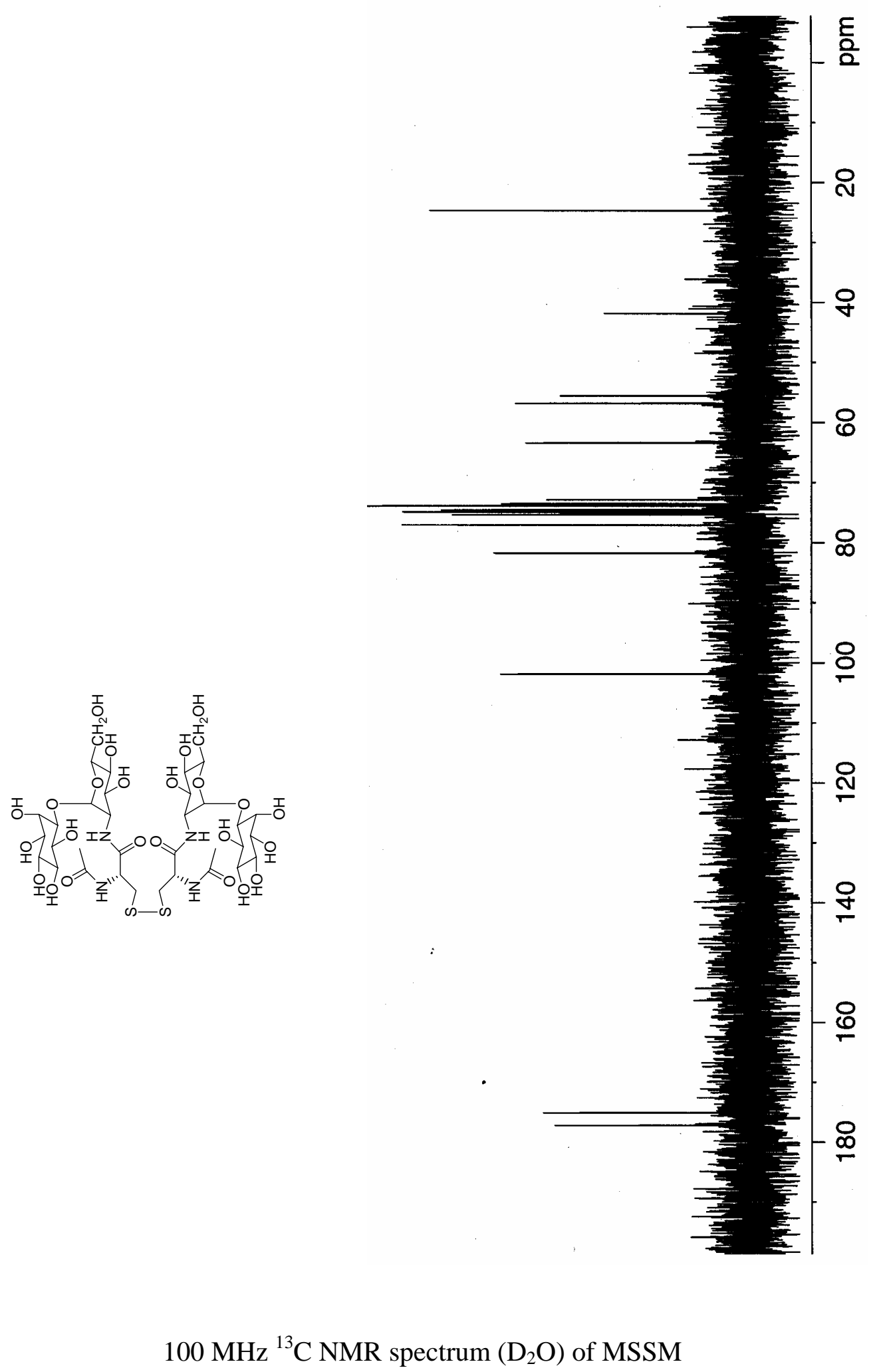




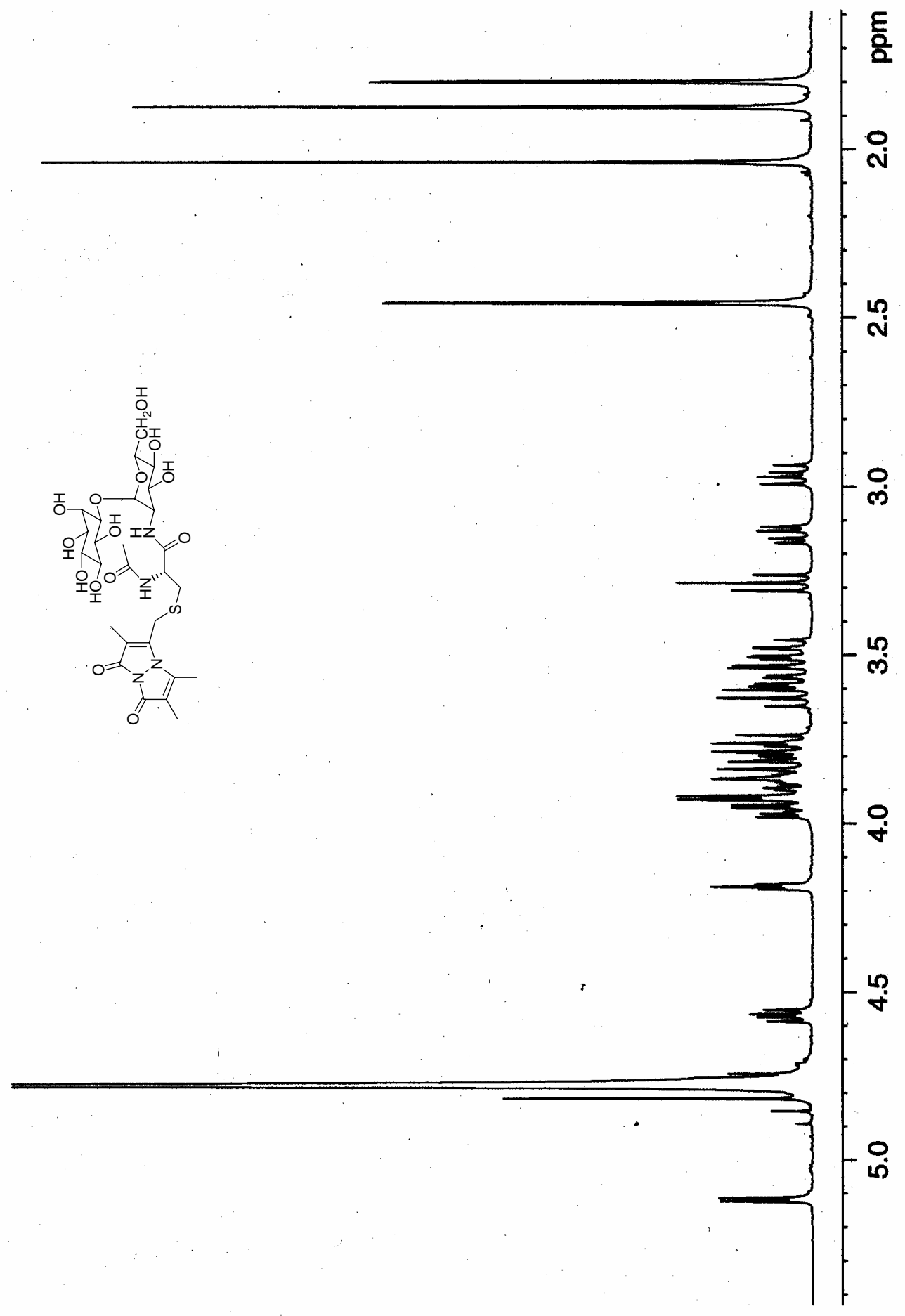

$400 \mathrm{MHz}{ }^{1} \mathrm{H}$ NMR spectrum $\left(\mathrm{D}_{2} \mathrm{O}\right)$ of $\mathrm{MSmB}$ 

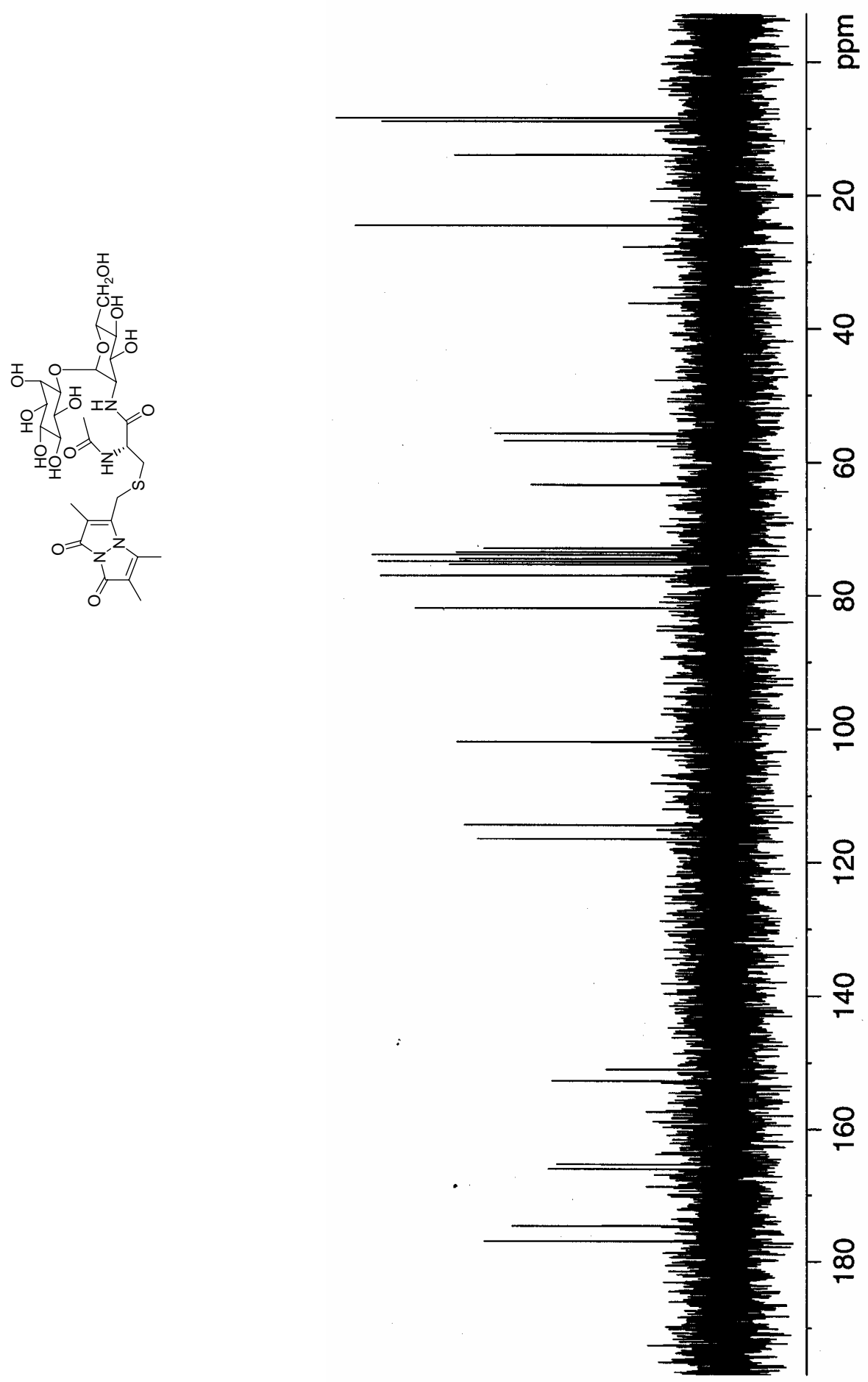

$100 \mathrm{MHz}{ }^{13} \mathrm{C}$ NMR spectrum $\left(\mathrm{D}_{2} \mathrm{O}\right)$ of $\mathrm{MSmB}$ 